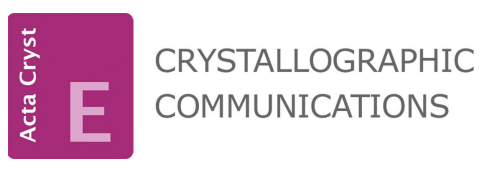

ISSN 2056-9890
Received 23 June 2020

Accepted 12 July 2020

Edited by H. Ishida, Okayama University, Japan

Keywords: cobalt(II); one-dimensional; zigzag; 4,4'-bipyridine; 2-hydroxybenzoate; crystal structure.

CCDC reference: 2015747

Supporting information: this article has supporting information at journals.iucr.org/e

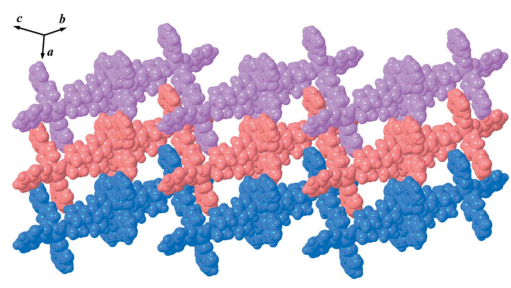

OPEN $\odot$ ACCESS

\section{Crystal structure of a novel one-dimensional zigzag chain-like cobalt(II) coordination polymer constructed from 4,4'-bipyridine and 2-hydroxy- benzoate ligands}

\author{
Thawanrat Saelim, ${ }^{\mathrm{a}, \mathrm{b}}$ Kittipong Chainok, $^{\mathrm{b}}$ Filip Kielar $^{\mathrm{c}}$ and Nanthawat Wannarit ${ }^{\mathrm{a}, \mathrm{b}}$
}

${ }^{a}$ Department of Chemistry, Faculty of Science and Technology, Thammasat University, Klong Luang, Pathum Thani 12121, Thailand, 'b Thammasat University Research Unit in Multifunctional Crystalline Materials and Applications (TUMCMA), Faculty of Science and Technology, Thammasat University, Klong Luang, Pathum Thani 12121, Thailand, and ' Department of Chemistry, Faculty of Science, Naresuan University, Phitsanulok 65000, Thailand. *Correspondence e-mail: nwan0110@tu.ac.th

A novel one-dimensional zigzag chain-like $\mathrm{Co}^{\mathrm{II}}$ coordination polymer constructed from 4,4'-bipyridine (4,4'-bpy) and 2-hydroxybenzoate (2-OHbenz) ligands, namely, catena-poly[[(4,4'-bipyridine- $\kappa N)(\mu$-2-hydroxybenzoato$\left.\kappa^{2} O: O^{\prime}\right)\left(2\right.$-hydroxybenzoato- $\left.\kappa^{2} O, O^{\prime}\right)$ cobalt(II)]- $\mu-4,4^{\prime}$-bipyridine- $\kappa^{2} N: N^{\prime}$-[aquahemi $\left(\mu-4,4^{\prime}\right.$-bipyridine- $\left.\kappa^{2} N: N^{\prime}\right)$ (2-hydroxybenzoato- $\kappa O$ (2-hydroxybenzoato$\left.\kappa^{2} O: O^{\prime}\right)$ cobalt(II)], $\left[\mathrm{Co}_{2}\left(\mathrm{C}_{7} \mathrm{H}_{5} \mathrm{O}_{3}\right)_{4}\left(\mathrm{C}_{10} \mathrm{H}_{8} \mathrm{~N}_{2}\right)_{2.5}\left(\mathrm{H}_{2} \mathrm{O}\right)\right]_{n}$, has been synthesized by reacting cobalt(II) nitrate trihydrate, $4,4^{\prime}$-bpy and 2 -hydroxybenzoic acid in a mixture of water and methanol at room temperature. There are two independent $\mathrm{Co}^{\mathrm{II}}$ centers, $\mathrm{Co} 1$ and $\mathrm{Co} 2$, in the asymmetric unit, revealing a distorted octahedral geometry with chromophore types of $\left[\mathrm{CoN}_{2} \mathrm{O}_{4}\right]$ and $\left[\mathrm{CoN}_{2} \mathrm{O}_{3} \mathrm{O}^{\prime}\right]$, respectively. The $\mathrm{Co} 1$ ions are doubly bridged by $2-\mathrm{OHbenz}$ ligands with syn-anti coordination mode, generating a dinuclear unit. The bridging 4,4'-bpy ligands connect these dinuclear units and the mononuclear $\mathrm{Co} 2$ chromophores, providing a one-dimensional alternating zigzag chain-like structure. In the crystal, intermolecular hydrogen bonds, $\mathrm{C}-\mathrm{H} \cdots \pi$ and $\pi-\pi$ stacking interactions are observed and these help to consolidate the packing. In addition, the physical properties of the title compound are reported.

\section{Chemical context}

The design and construction of new coordination polymers (CPs) is of current interest and attracts researchers in the fields of modern structural chemistry and materials science because of their potential applications in areas such as ionexchange, catalysis, sensors, magnetism, and non-linear optics (Dzhardimalieva \& Uflyand, 2017; Loukopoulos \& Kostakis, 2018; Horike et al., 2020). It is well known that the construction of CPs depends on a variety of factors such as the nature of metal ions and the organic ligands, the molar ratio of the reactants, and the reaction conditions e.g. reaction time, $\mathrm{pH}$, solvents, and temperature (Kitagawa et al., 2004; Noro et al., 2009). The structure-property relationships of hybrid polymeric materials with 4,4'-bipyridine (4,4'-bipy) have been studied intensively (Biradha et al., 2006; Khrizanforova et al., 2020). This is because $4,4^{\prime}$-bpy is a rigid molecule that can link the metal centers to form a network with well-defined structures and also support the stability of the structures through 
aromatic $\pi-\pi$ and $\mathrm{C}-\mathrm{H} \cdots \pi$ interactions (Kaes et al., 2000). Furthermore, many researchers incorporate carboxylatebased ligands for the construction of CPs, giving rise to frameworks with a variety of dimensions and topologies ( $\mathrm{Gu}$ et al., 2019; Horike et al., 2020). Benzoate and its derivatives have been widely used to construct the CPs because of the variety of their coordination modes, resulting in a variety of coordination geometries for the metal centers and interesting properties and applications of their CPs (Tong et al., 2000; Busskamp et al., 2007; Zhang et al., 2007; Song et al., 2009).

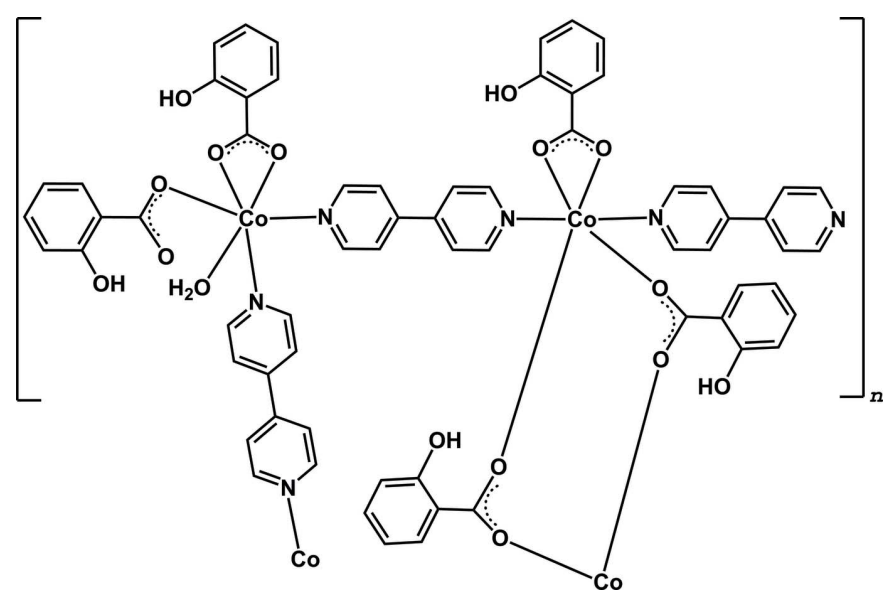

This work was undertaken as part of a search for new firstrow transition-metal coordination polymers constructed from $4,4^{\prime}$-bpy and carboxylate ligands. The $\mathrm{Co}^{\mathrm{II}}$ ion and hydroxybenzoate derivatives such as 2-hydroxybenzoate (2-OHbenz), 3-hydroxybenzoate (3-OHbenz) and 4-hydroxybenzoate (4OHbenz) have been utilized for this. As a result, a $\mathrm{Co}^{\text {II }}$ coordination polymer containing 4,4'-bpy and 2-OHbenz, $\left[\mathrm{Co}_{2}(2 \text {-OHbenz })_{4}\left(4,4^{\prime} \text {-bpy }\right)_{2.5}\left(\mathrm{H}_{2} \mathrm{O}\right)\right]_{n}$, with a novel $1 \mathrm{D}$ alternating zigzag chain-like structure has been successfully synthesized and characterized and its crystal structure has been determined. Herein, we report the synthesis and crystal structure and physical properties of this compound.

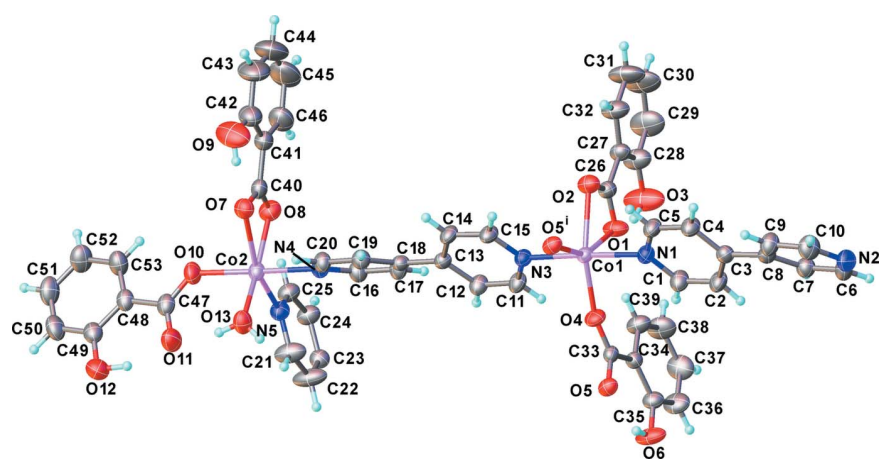

Figure 1

A segment of $\left[\mathrm{Co}_{2}(2-\mathrm{OHbenz})_{4}\left(4,4^{\prime}-\text {-bpy }\right)_{2.5}\left(\mathrm{H}_{2} \mathrm{O}\right)\right]_{n}$ with the atomlabeling scheme. Displacement ellipsoids are drawn at the $50 \%$ probability level. [Symmetry code: (i) $-x+1,-y,-z+1$.]
Table 1

Hydrogen-bond geometry $\left(\AA,^{\circ}\right)$.

$C g 4, C g 7$ and $C g 9$ are the centroids of the N4/C16-C20, C34-C39 and C48C53 rings, respectively.

\begin{tabular}{|c|c|c|c|c|}
\hline$D-\mathrm{H} \cdots A$ & $D-\mathrm{H}$ & $\mathrm{H} \cdots A$ & $D \cdots A$ & $D-\mathrm{H} \cdots A$ \\
\hline $\mathrm{O} 3-\mathrm{H} 3 \cdots \mathrm{O} 1$ & $0.85(3)$ & $1.77(3)$ & $2.553(3)$ & $153(4)$ \\
\hline $\mathrm{O} 6-\mathrm{H} 8 \cdots \mathrm{O} 5$ & $0.84(2)$ & $1.86(2)$ & $2.587(2)$ & $145(2)$ \\
\hline $\mathrm{O} 9-\mathrm{H} 13 \cdots \mathrm{O} 7$ & $0.84(2)$ & $1.79(2)$ & $2.568(3)$ & $152(4)$ \\
\hline $\mathrm{O} 12-\mathrm{H} 18 \cdots \mathrm{O} 11$ & $0.85(2)$ & $1.74(2)$ & $2.531(2)$ & $154(3)$ \\
\hline $\mathrm{O} 13-\mathrm{H} 26 \cdots \mathrm{O} 11$ & $0.84(1)$ & $1.82(2)$ & $2.625(2)$ & $160(2)$ \\
\hline $\mathrm{O} 13-\mathrm{H} 27 \cdots \mathrm{N} 2^{\mathrm{i}}$ & $0.83(2)$ & $2.05(2)$ & $2.860(3)$ & $167(2)$ \\
\hline $\mathrm{C} 25-\mathrm{H} 25 \cdots \mathrm{O} 8$ & 0.93 & 2.55 & $3.143(3)$ & 122 \\
\hline $\mathrm{C} 39-\mathrm{H} 39 \ldots \mathrm{O} 1$ & 0.93 & 2.38 & $3.271(3)$ & 160 \\
\hline $\mathrm{C} 5-\mathrm{H} 5 \cdots \mathrm{O} 12^{\mathrm{ii}}$ & 0.93 & 2.59 & $3.324(3)$ & 136 \\
\hline $\mathrm{C} 6-\mathrm{H} 6 \cdots \mathrm{O} 7^{\mathrm{iii}}$ & 0.93 & 2.48 & $3.300(3)$ & 147 \\
\hline $\mathrm{C} 12-\mathrm{H} 12 \cdots \mathrm{O}{ }^{\mathrm{iv}}$ & 0.93 & 2.58 & $3.173(3)$ & 122 \\
\hline $\mathrm{C} 15-\mathrm{H} 15 \cdots \mathrm{O}^{\mathrm{v}}$ & 0.93 & 2.51 & $3.292(3)$ & 142 \\
\hline $\mathrm{C} 2-\mathrm{H} 2 \cdots C g 9^{\mathrm{iii}}$ & 0.93 & 2.95 & $3.833(2)$ & 160 \\
\hline $\mathrm{C} 31-\mathrm{H} 31 \cdots C g 4^{\mathrm{vi}}$ & 0.93 & 2.85 & $3.681(4)$ & 149 \\
\hline $\mathrm{C} 51-\mathrm{H} 51 \cdots C g 7^{\mathrm{vii}}$ & 0.93 & 2.72 & $3.623(3)$ & 165 \\
\hline
\end{tabular}

Symmetry codes: (i) $-x+1,-y,-z+1$; (ii) $x, y-1, z+1$; (iii) $x+1, y-1, z+1$; (iv) $-x+1,-y+1,-z+1 ; \quad$ (v) $\quad x-1, y, z ; \quad$ (vi) $\quad-x,-y+1,-z+1$; $\quad$ (vii) $x-1, y+1, z-1$.

\section{Structural commentary}

The asymmetric unit consists of two independent $\mathrm{Co}^{\mathrm{II}}$ atoms, two and a half of 4,4'-bpy ligands, four 2-OHbenz ligands and one water molecule (Fig. 1). Both $\mathrm{Co}^{\mathrm{II}}$ centers exhibit a distorted octahedral geometry with $\left[\mathrm{CoN}_{2} \mathrm{O}_{4}\right]$ and $\left[\mathrm{CoN}_{2} \mathrm{O}_{3} \mathrm{O}^{\prime}\right]$ chromophores for $\mathrm{Co} 1$ and $\mathrm{Co} 2$, respectively (Fig. 2). The Co1 ion is coordinated by two $\mathrm{N}$ atoms from two $4,4^{\prime}$-bpy ligands with different monodentate and bridging coordination modes in a trans-configuration, and four $\mathrm{O}$ atoms

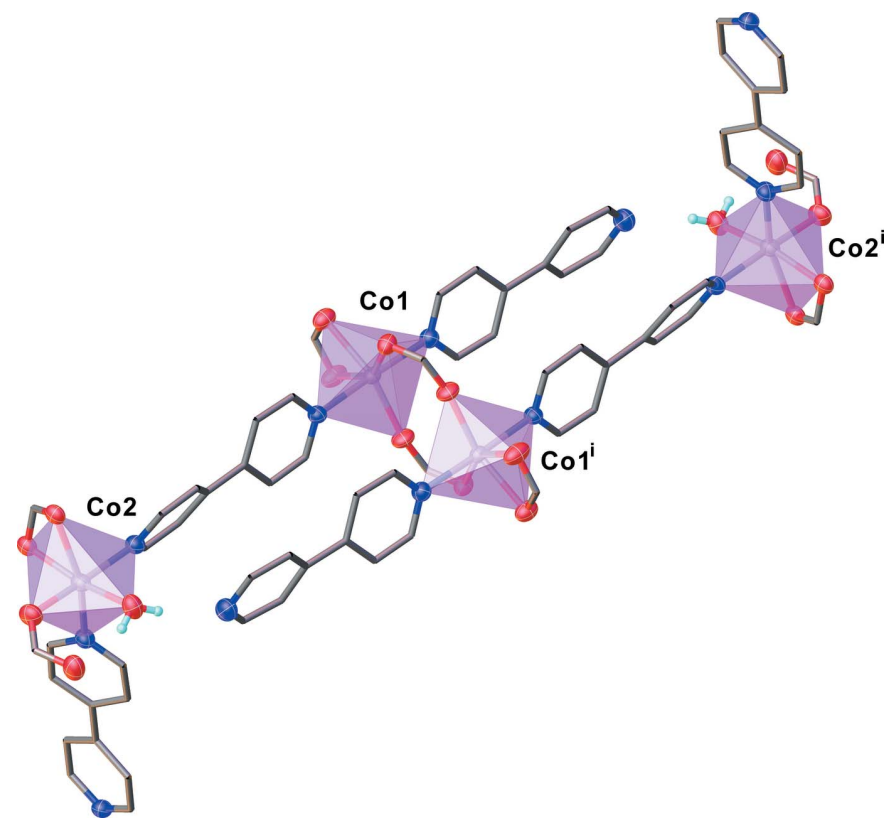

Figure 2

The coordination environments of $\mathrm{Co}^{\mathrm{II}}$ centers in the title compound. The hydrogen atoms on the aromatic rings and phenol ring on 2-OHbenz ligands have been omitted for clarity. [Symmetry code: (i) $-x+1,-y$, $-z+1$.] 
Table 2

Analysis of short ring interactions $(\AA)$.

$C g(\mathrm{I})$ and $C g(J)$ are the centroids of rings $I$ and $J ; C g I$ Perp is the perpendicular distance of $C g(I)$ on ring $J$ and slippage is the distance between $C g(I)$ and the perpendicular projection of $C g(J)$ on ring $I . C g 1, C g 2, C g 3, C g 4, C g 5, C g 6, C g 7$ and $C g 8$ are the centroids of the N1/C1-C5, N2/C6-C10, N3/C11-C15, N4/C16-C20, N5/C21-C25, C27-C32, C34-C39 and C41-C46 rings, respectively.

\begin{tabular}{|c|c|c|c|c|c|c|}
\hline$C g(I)$ & $C g(J)$ & Symmetry_Cg$(J)$ & $C g(I) \cdots C g(J)$ & CgI_Perp & CgJ_Perp & Slippage \\
\hline $\mathrm{Cg} 2$ & $\mathrm{Cg} 4$ & $-x+1,-y,-z+1$ & $3.6515(13)$ & $3.5063(9)$ & $3.5546(8)$ & 0.836 \\
\hline $\mathrm{Cg} 5$ & $\mathrm{Cg} 8$ & $-x,-y+2,-z$ & $4.0381(19)$ & $3.5840(10)$ & $3.5978(13)$ & 1.832 \\
\hline $\operatorname{Cg} 6$ & Cg8 & $x,-z+1,-z+1$ & $3.814(2)$ & $3.7674(13)$ & $3.7372(14)$ & 0.765 \\
\hline
\end{tabular}

from carboxylate groups of one terminal chelating and two bridging 2-OHbenz ligands, while the $\mathrm{Co} 2$ ion is bound to two $\mathrm{N}$ atoms of two $4,4^{\prime}$-bpy linkers in a cis-configuration, and four $\mathrm{O}$ atoms from carboxylate groups of two terminally monodentate and chelating 2-OHbenz ligands, and an aqua ligand. The $\mathrm{Co}-\mathrm{O}$ and $\mathrm{Co}-\mathrm{N}$ bond lengths fall in the ranges 2.0408 (14)-2.348 (15) and 2.1177 (16)-2.1568 (17) A, respectively. Two Co1 centers are doubly bridged by two bridging 2-OHbenz ligands with a syn-anti coordination mode to form a discrete dinuclear unit. The dinuclear units are connected to $\mathrm{Co} 2$ atoms by the bridging 4,4'-bpy ligands, providing a onedimensional zigzag chain-like structure along [101] (Fig. 3). The Co1 $\cdots$ Co $^{\mathrm{i}}$ [symmetry code: $\left.(\mathrm{i})=-x+1,-y,-z+1\right]$ and Co1 …Co2 distances are 4.099 (2) and 11.381 (2) Å, respectively.

Intramolecular hydrogen bonds (Table 1) comprise (i) $\mathrm{O}-$ $\mathrm{H}$. . O interactions formed by hydrogen donor atoms from the hydroxyl groups of 2-OHbenz and aqua ligands to oxygen acceptors in the carboxylate groups of the 2-OHbenz ligands and (ii) an $\mathrm{O}-\mathrm{H} \cdots \mathrm{N}$ interaction formed by a hydrogen-atom donor of the aqua ligand to an uncoordinated nitrogen acceptor atom in the terminal 4,4'-bpy ligand. The intramolecular $\pi-\pi$ stacking interactions involve the pyridyl rings of the $4,4^{\prime}$-bpy ligands, the intercentroid distances $C g 1 \cdots C g 3^{\mathrm{i}}$ and $C g 2 \cdots C g 4^{\mathrm{i}}$ being 3.965 (1) and 3.652 (1) $\AA$, respectively, where $C g 1, C g 2, C g 3$ and $C g 4$ are the centroids of the N1/C1C5, N2/C6-C10, N3/C11-C15 and N4/C16-C20 rings, respectively [symmetry code: (i) $-x+1,-y,-z+1$; Fig. 4].

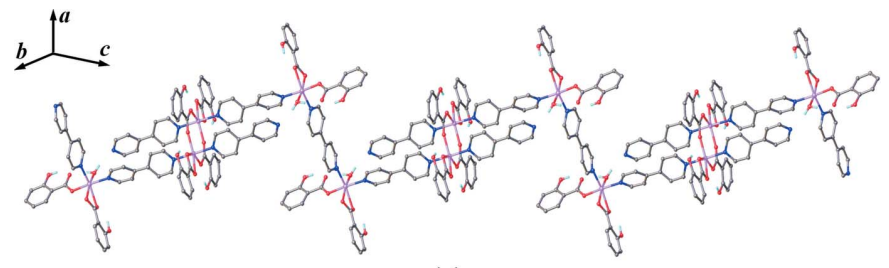

(a)

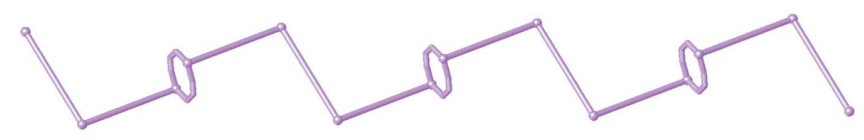

(b)

Figure 3

(a) View of the one-dimensional alternating zigzag chain-like structure and $(b)$ the schematic skeleton representing the topology of the title compound

\section{Supramolecular features}

The extended structure of the title compound is consolidated by hydrogen bonds and $\pi-\pi$ stacking and $\mathrm{C}-\mathrm{H} \cdots \pi$ interactions. The details of these weak interactions are summarized in Tables 1 and 2. The intermolecular interactions between the adjacent $1 \mathrm{D}$ zigzag chains are (i) $\mathrm{C}-\mathrm{H}$. . O hydrogen bonds between the benzene rings and hydroxyl groups of 2-OHbenz, (ii) $\pi-\pi$ stacking interactions between the bridging $4,4^{\prime}$-bpy and the terminal chelating 2-OHbenz and also between the phenyl rings of terminal chelating 2-OHbenz ligands, (iii) $\mathrm{C}-$ $\mathrm{H} \cdots \pi$ interactions between the $\mathrm{C}-\mathrm{H}$ of the terminal monodentate 2-OHbenz ligand and the pyridine ring of 4,4'-bpy ligands and (iv) $\mathrm{C}-\mathrm{H} \cdots \pi$ interactions between the terminal

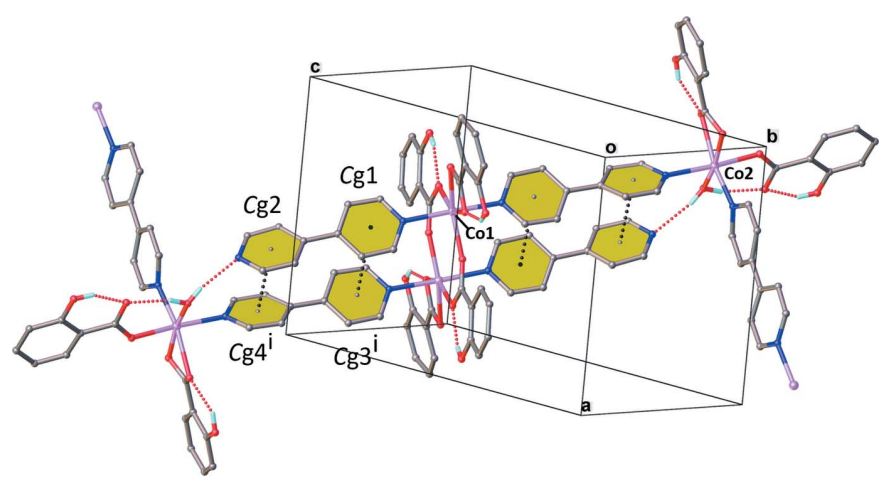

Figure 4

The intramolecular interactions in the title compound. The hydrogen atoms on aromatic rings have been omitted for clarity. $C \mathrm{~g} 1, C \mathrm{~g} 2, C \mathrm{~g} 3$ and $C \mathrm{~g} 4$ are the centroids of N1/C1-C5, N2/C6-C10, N3/C11-C15 and N4/ C16-C20 rings, respectively. [Symmetry code: (i) $-x+1,-y,-z+1$.]
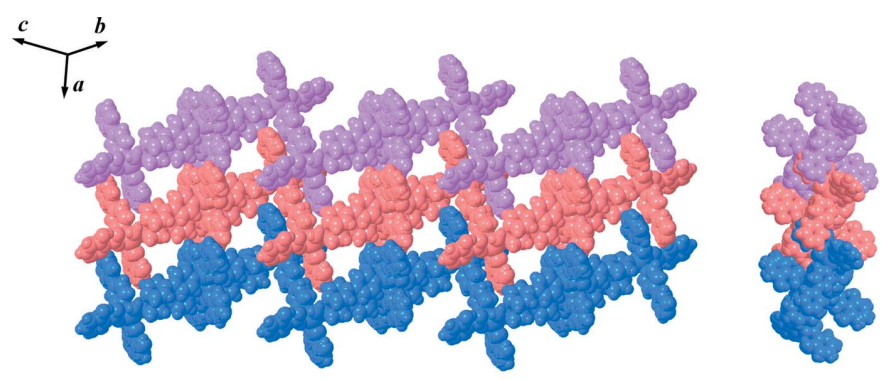

(a)

(b)

Figure 5

(a) Top and (b) side views of packing diagram in the (110) plane with a space-filling plot of adjacent one-dimensional zigzag chains of the title compound. 
chelating 2-OHbenz ligands. A packing diagram showing adjacent 1D zigzag chains in the (110) plane is shown in Fig. 5.

\section{Database survey}

No transition-metal CPs related to the title compound containing a $1 \mathrm{D}$ alternating zigzag chain-like structure have been reported. To the best of our knowledge, some related 1D chain-like $\mathrm{Co}^{\text {II }} \mathrm{CPs}$ containing $4,4^{\prime}$-bpy and benzoate or hydroxybenzoate derivatives have been reported with two different topologies. The 1D ladder-like structure topology has been found for two $\mathrm{Co}^{\mathrm{II}} \mathrm{CPs}$, namely, $\left[\mathrm{Co}_{2}\left(4,4^{\prime}-\mathrm{bpy}\right)_{3^{-}}\right.$ $\left.\left(\mathrm{H}_{2} \mathrm{O}\right)_{2}(\mathrm{phba})_{2}\right]\left(\mathrm{NO}_{3}\right)_{2} \cdot 4 \mathrm{H}_{2} \mathrm{O}, \quad(\mathrm{phba}=$ 4-hydroxybenzoate $)$ (MEDROC; Tong et al., 2000) and $\left[\mathrm{Co}_{2}\left(\mu_{2}-4,4^{\prime}-\mathrm{bpy}\right)_{2}\left(\mu_{2^{-}}\right.\right.$ benz $\left.)_{2}(\text { benz })_{2}\right]_{n}$, (benz = benzoate) (RIPSUF; Zhang et al., 2007), while a normal 1D zigzag chain-like structure has been found for $\left[\mathrm{Co}_{2}(\mathrm{benz})_{4}\left(4,4^{\prime}-\mathrm{bpy}\right)_{2}\right]_{n}$ (RIPSUF01; Song et al., 2009).

\section{Synthesis and crystallization}

A solution of 4,4'-bpy $(0.1562 \mathrm{~g}, 1.0 \mathrm{mmol})$ in $\mathrm{MeOH}(5 \mathrm{~mL})$ was slowly added into a solution of $\mathrm{Co}\left(\mathrm{NO}_{3}\right)_{2} \cdot 6 \mathrm{H}_{2} \mathrm{O}(0.2910 \mathrm{~g}$, $1.0 \mathrm{mmol})$ in a $4: 1$ mixture of methanol and water $(10 \mathrm{~mL})$. The resulting solution was stirred for $20 \mathrm{~min}$. Next, a solution of 2-OHbenzH $(0.1382 \mathrm{~g}, 1.0 \mathrm{mmol})$ in methanol $(5 \mathrm{~mL})$ was slowly added dropwise and stirred over a period of $15 \mathrm{~min}$. After that, the mixture was filtered. The filtered solution was left to stand without disturbance and allowed to slowly evaporate in the air. After five days, red crystals suitable for single crystal X-ray diffraction were obtained $[56.18 \%$ yield based on cobalt(II) salt]. Elemental analysis; calculated for $\mathrm{C}_{53} \mathrm{H}_{42} \mathrm{Co}_{2} \mathrm{~N}_{5} \mathrm{O}_{13}$ : C 59.06, H 4.21, N 6.50\%; found: C 59.14, $\mathrm{H}$ 3.99, N 6.41\%. IR (KBr, $\left.v / \mathrm{cm}^{-1}\right): 3087 s, 1595 s, 1485 s, 1460 s$, $1459 s, 1413 m, 1389 s, 1359 s, 1308 w, 1252 m, 1218 m, 1143 w$, $1068 w, 1029 w, 871 w, 814 s, 749 s, 701 w, 671 w, 633 w, 530 w$.

The IR spectrum of the title compound (see Fig. S1 in the supporting information) shows a characteristic broad peak centered at $3087 \mathrm{~cm}^{-1}$, which is assigned to $\mathrm{OH}$ stretching vibrations of the water molecule and the hydroxyl groups of 2-OHbenz. Strong and sharp peaks at 1595 and $1485 \mathrm{~cm}^{-1}$ can be assigned as the asymmetric and symmetric $\mathrm{COO}^{-}$ stretching vibrations of the chelating 2-OHbenz ligands, respectively. Peaks in the region of $600-1000 \mathrm{~cm}^{-1}$ are assigned to $\mathrm{CH}$ bending of the aromatic rings in the ligands (Zhu et al., 2016).

The solid-state electronic spectrum of the title compound (Fig. S2) shows two broad bands in the visible region with the main peak centered about $515 \mathrm{~nm}(19.42 \mathrm{kK})$, which can be assigned to the $v_{3}:{ }^{4} T_{1 g} \rightarrow{ }^{4} T_{1 g}(P)$ transition. There is a small peak as a shoulder at around $655 \mathrm{~nm}(15.27 \mathrm{kK})$, assigned to the $\nu_{2}:{ }^{4} T_{1 g} \rightarrow{ }^{4} A_{2 g}$ transition and a broad band centered about $1095 \mathrm{~nm}(13.24 \mathrm{kK})$, which can be assigned to the $v_{1}$ : ${ }^{4} T_{1 g} \rightarrow{ }^{4} T_{2 g}$ transition. The characteristic bands of this electronic spectrum correspond to a distorted octahedral geometry for $\mathrm{Co}^{\mathrm{II}}$ compounds as confirmed by the X-ray structure (Piromchom et al., 2014).
Table 3

Experimental details.

\begin{tabular}{|c|c|}
\hline \multicolumn{2}{|l|}{ Crystal data } \\
\hline Chemical formula & {$\left[\mathrm{Co}_{2}\left(\mathrm{C}_{7} \mathrm{H}_{5} \mathrm{O}_{3}\right)_{4}\left(\mathrm{C}_{10} \mathrm{H}_{8} \mathrm{~N}_{2}\right)_{2.5}\left(\mathrm{H}_{2} \mathrm{O}\right)\right]$} \\
\hline$M_{\mathrm{r}}$ & 1074.77 \\
\hline Crystal system, space group & Triclinic, $P \overline{1}$ \\
\hline Temperature (K) & 296 \\
\hline$a, b, c(\AA)$ & $\begin{array}{l}10.8832(18), 11.4742(19), \\
\quad 19.905(3)\end{array}$ \\
\hline$\alpha, \beta, \gamma\left({ }^{\circ}\right)$ & $74.295(5), 89.791(5), 88.502(6)$ \\
\hline$V\left(\AA^{3}\right)$ & $2392.0(7)$ \\
\hline$Z$ & 2 \\
\hline Radiation type & Мо $K \alpha$ \\
\hline$\mu\left(\mathrm{mm}^{-1}\right)$ & 0.77 \\
\hline Crystal size $(\mathrm{mm})$ & $0.32 \times 0.24 \times 0.2$ \\
\hline \multicolumn{2}{|l|}{ Data collection } \\
\hline Diffractometer & Bruker D8 Quest CMOS Photon II \\
\hline Absorption correction & $\begin{array}{l}\text { Multi-scan (SADABS; Bruker, } \\
\text { 2016) }\end{array}$ \\
\hline$T_{\min }, T_{\max }$ & $0.677,0.746$ \\
\hline $\begin{array}{l}\text { No. of measured, independent and } \\
\text { observed }[I>2 \sigma(I)] \text { reflections }\end{array}$ & $60283,9771,7780$ \\
\hline$R_{\text {int }}$ & 0.042 \\
\hline$(\sin \theta / \lambda)_{\max }\left(\AA^{-1}\right)$ & 0.625 \\
\hline \multicolumn{2}{|l|}{ Refinement } \\
\hline$R\left[F^{2}>2 \sigma\left(F^{2}\right)\right], w R\left(F^{2}\right), S$ & $0.035,0.088,1.05$ \\
\hline No. of reflections & 9771 \\
\hline No. of parameters & 682 \\
\hline No. of restraints & 6 \\
\hline $\mathrm{H}$-atom treatment & $\begin{array}{l}\mathrm{H} \text { atoms treated by a mixture of } \\
\text { independent and constrained } \\
\text { refinement }\end{array}$ \\
\hline$\Delta \rho_{\max }, \Delta \rho_{\min }\left(\mathrm{e} \AA^{-3}\right)$ & $0.43,-0.30$ \\
\hline
\end{tabular}

Computer programs: APEX3 and SAINT (Bruker, 2016), SHELXT (Sheldrick, 2015a), SHELXL (Sheldrick, 2015b) and OLEX2 (Dolomanov et al., 2009).

The PXRD pattern of the title compound (Fig. S3) was used to check the phase purity of the bulk sample in the solid state. The measured PXRD pattern of the title compound closely matches the simulated pattern generated from the singlecrystal X-ray diffraction data, confirming the title compound is pure.

The TGA curve shown in (Fig. S4) demonstrates the thermal stability of the title compound up to $160^{\circ} \mathrm{C}$. The first weight-loss step of $27.37 \%$ is observed from 160 to $277^{\circ} \mathrm{C}$ and can be attributed to the loss of coordinated water and two 2-OHbenz molecules. The next step weight-loss step of $25.7 \%$ observed from 277 to $356^{\circ} \mathrm{C}$ corresponds to the loss of a coordinated 2-OHbenz molecule. Finally, the weight loss of about $36.33 \%$ from 356 to $520^{\circ} \mathrm{C}$ can be assigned to the removal of two and half of the 4,4'-bpy ligands. The residual product is assumed to be $\mathrm{CoO}$.

The solid-state photoluminescent properties of the title compound and free ligands were investigated at room temperature. As shown in Fig. S5, the emission spectra of the free ligands 4,4'-bpy and 2-OHbenzH $\left(\lambda_{\mathrm{ex}}=340 \mathrm{~nm}\right)$ exhibit strong emission bands at 425 and $439 \mathrm{~nm}$, respectively. However, no detectable emission can be observed for the title compound $\left(\lambda_{\mathrm{ex}}=340 \mathrm{~nm}\right)$. This complete PL quenching is the result of the low energy $d-d$ transitions in the partially filled metal ion centers found for $\mathrm{Co}^{\mathrm{II}}$ compounds described above and reported elsewhere (Yang et al., 2012; Zhu et al., 2014). 


\section{Refinement}

Crystal data, data collection and structure refinement details are summarized in Table 3. C-bound $\mathrm{H}$ atoms were positioned geometrically and refined using a riding model, with $\mathrm{C}-\mathrm{H}=$ $0.93 \AA$ and the $U_{\text {iso }}(\mathrm{H})=1.2 U_{\text {eq }}(\mathrm{C})$. O-bound $\mathrm{H}$ atoms were located in a difference electron-density map, and were refined with bond-length restraints of $\mathrm{O}-\mathrm{H}=0.84$ (1) $\AA$.

\section{Acknowledgements}

The authors are grateful to Faculty of Science and Technology, Thammasat University for funds to purchase the X-ray diffractometer.

\section{Funding information}

Funding for this research was provided by: Faculty of Science and Technology, Thammasat University (contract No. SciGR7/ 2563 to N. Wannarit); Thammasat University Research Unit in Multifunctional Crystalline Materials and Applications (TUMCMA) (grant to K. Chainok, N. Wannarit).

\section{References}

Biradha, K., Sarkar, M. \& Rajput, L. (2006). Chem. Commun. pp. 4169-4179.

Bruker (2016). APEX2, SAINT and $S A D A B S$. Bruker AXS Inc., Madison, Wisconsin, USA.

Busskamp, H., Deacon, G. B., Hilder, M., Junk, P. C., Kynast, U. H., Lee, W. W. \& Turner, D. R. (2007). CrystEngComm, 9, 394-411.

Dolomanov, O. V., Bourhis, L. J., Gildea, R. J., Howard, J. A. K. \& Puschmann, H. (2009). J. Appl. Cryst. 42, 339-341.
Dzhardimalieva, G. I. \& Uflyand, I. E. (2017). RSC Adv. 7, 4224242288.

Gu, J., Wen, M., Cai, Y., Shi, Z., Nesterov, D. S., Kirillova, M. V. \& Kirillov, A. M. (2019). Inorg. Chem. 58, 5875-5885.

Horike, S., Nagarkar, S. S., Ogawa, T. \& Kitagawa, S. (2020). Angew. Chem. Int. Ed. 59, 6652-6664.

Kaes, C., Katz, A. \& Hosseini, M. W. (2000). Chem. Rev. 100, $3553-$ 3590.

Khrizanforova, V., Shekurov, R., Miluykov, V., Khrizanforov, M., Bon, V., Kaskel, S., Gubaidullin, A., Sinyashin, O. \& Budnikova, Y. (2020). Dalton Trans. 49, 2794-2802.

Kitagawa, S., Kitaura, R. \& Noro, S.-I. (2004). Angew. Chem. Int. Ed. 43, 2334-2375.

Loukopoulos, E. \& Kostakis, G. E. (2018). J. Coord. Chem. 71, 371410.

Noro, S.-I., Kitagawa, S., Akutagawa, T. \& Nakamura, T. (2009). Prog. Polym. Sci. 34, 240-279.

Piromchom, J., Wannarit, N., Boonmak, J., Pakawatchai, C. \& Youngme, S. (2014). Inorg. Chem. Commun. 40, 59-61.

Sheldrick, G. M. (2015a). Acta Cryst. A71, 3-8.

Sheldrick, G. M. (2015b). Acta Cryst. C71, 3-8.

Song, Y. J., Kwak, H., Lee, Y. M., Kim, S. H., Lee, S. H., Park, B. K., Jun, J. Y., Yu, S. M., Kim, C., Kim, S.-J. \& Kim, Y. (2009). Polyhedron, 28, 1241-1252.

Tong, M.-L., Chen, H.-J. \& Chen, X.-M. (2000). Inorg. Chem. 39, 2235-2238.

Yang, J., Shen, L., Yang, G.-W., Li, Q.-Y., Shen, W., Jin, J.-N., Zhao, J.-J. \& Dai, J. (2012). J. Solid State Chem. 186, 124-133.

Zhang, Z.-X., Li, Y., Li, K.-C., Song, W.-D. \& Li, Q.-S. (2007). Inorg. Chem. Commun. 10, 1276-1280.

Zhu, D., Tian, H., Li, F., Xie, J., Zhang, P., Zou, J., Zhao, L., Zhang, F., Yang, G. \& Li, Q. (2014). J. Inorg. Organomet. Polym. 24, $1103-$ 1109.

Zhu, W.-G., Lin, C.-J., Zheng, Y.-Q. \& Zhu, H.-L. (2016). Transition Met. Chem. 41, 87-96. 


\section{supporting information}

Acta Cryst. (2020). E76, 1302-1306 [https://doi.org/10.1107/S2056989020009482]

Crystal structure of a novel one-dimensional zigzag chain-like cobalt(II) coordination polymer constructed from 4,4'-bipyridine and 2-hydroxybenzoate ligands

\section{Thawanrat Saelim, Kittipong Chainok, Filip Kielar and Nanthawat Wannarit}

Computing details

Data collection: APEX3 (Bruker, 2016); cell refinement: SAINT (Bruker, 2016); data reduction: SAINT (Bruker, 2016); program(s) used to solve structure: SHELXT (Sheldrick, 2015a); program(s) used to refine structure: SHELXL (Sheldrick, 2015b); molecular graphics: OLEX2 (Dolomanov et al., 2009); software used to prepare material for publication: OLEX2 (Dolomanov et al., 2009).

catena-Poly $\left[\left[\left(4,4^{\prime}\right.\right.\right.$-bipyridine- $\left.\kappa N\right)\left(\mu\right.$-2-hydroxybenzoato- $\left.\kappa^{2} O: O^{\prime}\right)\left(2\right.$-hydroxybenzoato- $\left.\kappa^{2} O, O^{\prime}\right)$ cobalt(II)]- $\mu-4,4^{\prime}$ bipyridine- $\kappa^{2} N: N^{\prime}$-[aquahemi $\left(\mu-4,4^{\prime}\right.$-bipyridine- $\left.\kappa^{2} N: N^{\prime}\right)(2$-hydroxybenzoato- $\kappa O)$ (2-hydroxybenzoato-

$\left.\kappa^{2} O, O^{\prime}\right)$ cobalt $\left.(\mathrm{II})\right]$

Crystal data

$\left[\mathrm{Co}_{2}\left(\mathrm{C}_{7} \mathrm{H}_{5} \mathrm{O}_{3}\right)_{4}\left(\mathrm{C}_{10} \mathrm{H}_{8} \mathrm{~N}_{2}\right)_{2.5}\left(\mathrm{H}_{2} \mathrm{O}\right)\right]$

$$
Z=2
$$

$M_{r}=1074.77$

$F(000)=1106$

Triclinic, $P \overline{1}$

$a=10.8832(18) \AA$

$D_{\mathrm{x}}=1.492 \mathrm{Mg} \mathrm{m}^{-3}$

$b=11.4742(19) \AA$

$c=19.905(3) \AA$

$\alpha=74.295(5)^{\circ}$

$\beta=89.791(5)^{\circ}$

$\gamma=88.502(6)^{\circ}$

Mo $K \alpha$ radiation, $\lambda=0.71073 \AA$

Cell parameters from 9918 reflections

$\theta=3.1-28.2^{\circ}$

$\mu=0.77 \mathrm{~mm}^{-1}$

$T=296 \mathrm{~K}$

$V=2392.0(7) \AA^{3}$

Block, red

$0.32 \times 0.24 \times 0.2 \mathrm{~mm}$

\section{Data collection}

Bruker D8 Quest CMOS Photon II diffractometer

Radiation source: sealed x-ray tube, Mo

Graphite monochromator

Detector resolution: 7.39 pixels $\mathrm{mm}^{-1}$

$\omega$ and $\varphi$ scans

Absorption correction: multi-scan

(SADABS; Bruker, 2016)

$T_{\text {min }}=0.677, T_{\max }=0.746$

60283 measured reflections

9771 independent reflections

7780 reflections with $I>2 \sigma(I)$

$R_{\text {int }}=0.042$

$\theta_{\text {max }}=26.4^{\circ}, \theta_{\min }=3.0^{\circ}$

$h=-13 \rightarrow 13$

$k=-14 \rightarrow 14$

$l=-24 \rightarrow 24$

Refinement

Refinement on $F^{2}$

Least-squares matrix: full

$w R\left(F^{2}\right)=0.088$

$R\left[F^{2}>2 \sigma\left(F^{2}\right)\right]=0.035$

$S=1.05$

9771 reflections 
682 parameters

6 restraints

Primary atom site location: dual

Hydrogen site location: mixed

$\mathrm{H}$ atoms treated by a mixture of independent and constrained refinement

$$
\begin{aligned}
& w=1 /\left[\sigma^{2}\left(F_{\mathrm{o}}^{2}\right)+(0.0419 P)^{2}+0.8816 P\right] \\
& \text { where } P=\left(F_{\mathrm{o}}^{2}+2 F_{\mathrm{c}}^{2}\right) / 3 \\
& (\Delta / \sigma)_{\max }<0.001 \\
& \Delta \rho_{\max }=0.43 \mathrm{e} \AA^{-3} \\
& \Delta \rho_{\min }=-0.29 \mathrm{e} \AA^{-3}
\end{aligned}
$$

\section{Special details}

Geometry. All esds (except the esd in the dihedral angle between two 1.s. planes) are estimated using the full covariance

\begin{tabular}{|c|c|c|c|c|}
\hline & $x$ & $y$ & $z$ & $U_{\text {iso }} * / U_{\text {eq }}$ \\
\hline Co1 & $0.38320(2)$ & $0.10363(2)$ & $0.54244(2)$ & $0.02725(7)$ \\
\hline $\mathrm{Co} 2$ & $0.06977(2)$ & $0.72632(2)$ & $0.02018(2)$ & $0.03175(8)$ \\
\hline $\mathrm{O} 1$ & 0.40605 (13) & $0.25879(14)$ & $0.58404(9)$ & $0.0484(4)$ \\
\hline $\mathrm{O} 2$ & $0.23200(13)$ & $0.16721(13)$ & $0.59633(8)$ & $0.0409(3)$ \\
\hline $\mathrm{O} 3$ & $0.4298(2)$ & $0.4553(2)$ & $0.61905(15)$ & $0.0980(9)$ \\
\hline H3 & $0.443(4)$ & $0.398(2)$ & 0.6003 (19) & $0.121(14)^{*}$ \\
\hline $\mathrm{O} 4$ & $0.55720(12)$ & $0.11808(13)$ & $0.50300(8)$ & $0.0402(3)$ \\
\hline $\mathrm{O} 5$ & $0.72507(12)$ & $0.03072(12)$ & $0.47519(7)$ & $0.0344(3)$ \\
\hline O6 & $0.93656(14)$ & 0.12657 (17) & $0.47886(11)$ & $0.0602(5)$ \\
\hline H8 & $0.888(2)$ & $0.077(2)$ & $0.4705(14)$ & $0.071(9)^{*}$ \\
\hline $\mathrm{O} 7$ & $-0.11751(13)$ & $0.67237(13)$ & $0.06159(7)$ & $0.0406(3)$ \\
\hline $\mathrm{O} 8$ & $-0.03026(13)$ & $0.83546(13)$ & $0.07254(8)$ & $0.0438(4)$ \\
\hline O9 & $-0.3306(2)$ & $0.6158(2)$ & $0.11428(13)$ & $0.0875(7)$ \\
\hline H13 & $-0.2608(17)$ & $0.610(3)$ & $0.0971(18)$ & $0.106(13)^{*}$ \\
\hline $\mathrm{O} 10$ & $-0.00286(14)$ & $0.81713(14)$ & $-0.07657(7)$ & $0.0454(4)$ \\
\hline O11 & $0.10789(16)$ & $0.73590(15)$ & $-0.14789(8)$ & $0.0543(4)$ \\
\hline $\mathrm{O} 12$ & $0.08458(17)$ & $0.81806(18)$ & $-0.27880(9)$ & $0.0592(5)$ \\
\hline H18 & $0.107(3)$ & $0.775(2)$ & $-0.2386(9)$ & $0.088(11)^{*}$ \\
\hline $\mathrm{O} 13$ & $0.13809(16)$ & $0.59112(14)$ & $-0.02174(8)$ & $0.0441(4)$ \\
\hline $\mathrm{H} 26$ & $0.135(3)$ & $0.623(2)$ & $-0.0648(6)$ & $0.078(10) *$ \\
\hline $\mathrm{H} 27$ & 0.2003 (16) & $0.549(2)$ & $-0.0066(13)$ & $0.063(9)^{*}$ \\
\hline N1 & $0.44634(15)$ & $-0.01847(15)$ & $0.63825(8)$ & $0.0339(4)$ \\
\hline $\mathrm{N} 2$ & $0.66774(18)$ & $-0.43591(18)$ & $0.94863(9)$ & $0.0474(5)$ \\
\hline N3 & $0.32399(14)$ & $0.22088(14)$ & $0.44421(8)$ & $0.0311(3)$ \\
\hline N4 & $0.13327(15)$ & $0.61430(14)$ & $0.11989(8)$ & $0.0332(4)$ \\
\hline N5 & $0.22979(15)$ & $0.83133(15)$ & $0.00504(9)$ & $0.0357(4)$ \\
\hline $\mathrm{C} 1$ & $0.56246(19)$ & $-0.0201(2)$ & $0.66090(11)$ & $0.0418(5)$ \\
\hline H1 & 0.616518 & 0.035341 & 0.634347 & $0.050 *$ \\
\hline $\mathrm{C} 2$ & 0.60535 (19) & $-0.0993(2)$ & $0.72121(11)$ & $0.0417(5)$ \\
\hline $\mathrm{H} 2$ & 0.687050 & -0.097234 & 0.734268 & $0.050 *$ \\
\hline $\mathrm{C} 3$ & $0.52771(18)$ & $-0.18256(17)$ & $0.76293(10)$ & $0.0331(4)$ \\
\hline $\mathrm{C} 4$ & $0.40701(18)$ & $-0.18020(19)$ & $0.73970(11)$ & $0.0393(5)$ \\
\hline $\mathrm{H} 4$ & 0.350822 & -0.233833 & 0.765656 & $0.047 *$ \\
\hline
\end{tabular}
matrix. The cell esds are taken into account individually in the estimation of esds in distances, angles and torsion angles; correlations between esds in cell parameters are only used when they are defined by crystal symmetry. An approximate (isotropic) treatment of cell esds is used for estimating esds involving l.s. planes.

Fractional atomic coordinates and isotropic or equivalent isotropic displacement parameters $\left(\AA^{2}\right)$ 


\begin{tabular}{|c|c|c|c|c|}
\hline $\mathrm{C} 5$ & $0.37092(18)$ & -0.09847 (19) & $0.67830(11)$ & $0.0393(5)$ \\
\hline H5 & 0.289795 & -0.098811 & 0.663857 & $0.047^{*}$ \\
\hline C6 & $0.7144(2)$ & $-0.3259(2)$ & 0.92367 (11) & $0.0451(5)$ \\
\hline H6 & 0.780326 & -0.305313 & 0.947374 & $0.054 *$ \\
\hline $\mathrm{C} 7$ & $0.67096(19)$ & $-0.24166(19)$ & $0.86514(11)$ & $0.0386(5)$ \\
\hline $\mathrm{H} 7$ & 0.707240 & -0.166564 & 0.850264 & $0.046^{*}$ \\
\hline C8 & $0.57241(18)$ & $-0.26897(18)$ & $0.82815(10)$ & $0.0342(4)$ \\
\hline C9 & $0.5219(2)$ & $-0.3816(2)$ & 0.85489 (11) & $0.0444(5)$ \\
\hline H9A & 0.454541 & -0.403962 & 0.833014 & $0.053^{*}$ \\
\hline $\mathrm{C} 10$ & $0.5716(2)$ & $-0.4605(2)$ & $0.91394(12)$ & $0.0514(6)$ \\
\hline H10 & 0.535930 & -0.535569 & 0.930657 & $0.062^{*}$ \\
\hline C11 & $0.39719(18)$ & $0.30579(18)$ & $0.40608(11)$ & $0.0373(5)$ \\
\hline H11 & 0.475641 & 0.312109 & 0.422913 & $0.045^{*}$ \\
\hline $\mathrm{C} 12$ & $0.36219(18)$ & $0.38385(18)$ & $0.34346(11)$ & $0.0367(5)$ \\
\hline H12 & 0.416736 & 0.440794 & 0.318790 & $0.044 *$ \\
\hline C13 & $0.24535(17)$ & $0.37796(17)$ & $0.31686(10)$ & $0.0299(4)$ \\
\hline $\mathrm{C} 14$ & $0.16882(17)$ & $0.29091(18)$ & $0.35696(10)$ & $0.0365(5)$ \\
\hline H14 & 0.089433 & 0.283600 & 0.341728 & $0.044 *$ \\
\hline $\mathrm{C} 15$ & 0.21083 & $0.21556(19)$ & $0.41926(10)$ & $0.0366(5)$ \\
\hline H15 & 0.158001 & 0.158196 & 0.445261 & $0.044 *$ \\
\hline $\mathrm{C} 16$ & 0.20919 (19) & $0.65071(18)$ & $0.16245(10)$ & $0.0376(5)$ \\
\hline H16 & 0.237771 & 0.729246 & 0.148137 & $0.045^{*}$ \\
\hline C17 & $0.24722(19)$ & $0.57804(18)$ & $0.22630(10)$ & $0.0372(5)$ \\
\hline H17 & 0.300167 & 0.607670 & 0.253975 & $0.045^{*}$ \\
\hline $\mathrm{C} 18$ & $0.20611(16)$ & $0.45990(17)$ & $0.24934(10)$ & $0.0301(4)$ \\
\hline C19 & $0.12639(18)$ & $0.42283(18)$ & $0.20528(10)$ & $0.0345(4)$ \\
\hline H19 & 0.095861 & 0.344987 & 0.218331 & $0.041^{*}$ \\
\hline $\mathrm{C} 20$ & $0.09266(18)$ & $0.50133(18)$ & $0.14235(10)$ & $0.0361(4)$ \\
\hline $\mathrm{H} 20$ & 0.038814 & 0.474499 & 0.113901 & $0.043 *$ \\
\hline $\mathrm{C} 21$ & $0.3388(2)$ & $0.7903(2)$ & $-0.01008(16)$ & $0.0613(7)$ \\
\hline $\mathrm{H} 21$ & 0.343265 & 0.715188 & -0.019510 & $0.074^{*}$ \\
\hline $\mathrm{C} 22$ & $0.4454(2)$ & $0.8530(2)$ & $-0.01252(17)$ & $0.0668(8)$ \\
\hline $\mathrm{H} 22$ & 0.519350 & 0.819901 & -0.023310 & $0.080^{*}$ \\
\hline $\mathrm{C} 23$ & $0.44329(18)$ & $0.96475(18)$ & $0.00095(11)$ & $0.0361(4)$ \\
\hline $\mathrm{C} 24$ & 0.32980 (19) & $1.00692(19)$ & $0.01651(12)$ & $0.0419(5)$ \\
\hline $\mathrm{H} 24$ & 0.322945 & 1.081478 & 0.026449 & $0.050^{*}$ \\
\hline $\mathrm{C} 25$ & $0.22646(19)$ & 0.93955 (19) & $0.01745(12)$ & $0.0416(5)$ \\
\hline $\mathrm{H} 25$ & 0.151031 & 0.971218 & 0.027200 & $0.050^{*}$ \\
\hline $\mathrm{C} 26$ & $0.29583(18)$ & $0.25023(18)$ & $0.60563(10)$ & $0.0342(4)$ \\
\hline $\mathrm{C} 27$ & $0.24654(18)$ & $0.33984(19)$ & $0.64101(11)$ & $0.0382(5)$ \\
\hline $\mathrm{C} 28$ & $0.3152(2)$ & $0.4384(2)$ & $0.64491(15)$ & $0.0584(7)$ \\
\hline $\mathrm{C} 29$ & $0.2655(3)$ & $0.5232(3)$ & $0.67603(19)$ & $0.0848(10)$ \\
\hline $\mathrm{H} 29$ & 0.309833 & 0.590962 & 0.676996 & $0.102 *$ \\
\hline $\mathrm{C} 30$ & $0.1514(3)$ & $0.5073(3)$ & $0.7053(2)$ & $0.0926(11)$ \\
\hline $\mathrm{H} 30$ & 0.119079 & 0.563885 & 0.726726 & $0.111^{*}$ \\
\hline $\mathrm{C} 31$ & $0.0841(3)$ & $0.4094(3)$ & $0.7035(2)$ & $0.0870(11)$ \\
\hline H31 & 0.006936 & 0.398807 & 0.724094 & $0.104 *$ \\
\hline C32 & $0.1313(2)$ & $0.3262(2)$ & $0.67084(14)$ & $0.0585(7)$ \\
\hline
\end{tabular}




$\begin{array}{lllll}\text { H32 } & 0.084907 & 0.260227 & 0.668929 & 0.070^{*} \\ \text { C33 } & 0.67051(16) & 0.11563(17) & 0.49469(9) & 0.0278(4) \\ \text { C34 } & 0.74627(17) & 0.21332(16) & 0.50758(9) & 0.0289(4) \\ \text { C35 } & 0.87402(18) & 0.21315(18) & 0.50003(11) & 0.0368(5) \\ \text { C36 } & 0.9411(2) & 0.3034(2) & 0.51642(14) & 0.0539(6) \\ \text { H36 } & 1.026195 & 0.303308 & 0.511912 & 0.065^{*} \\ \text { C37 } & 0.8821(2) & 0.3925(2) & 0.53918(14) & 0.0581(7) \\ \text { H37 } & 0.927865 & 0.451984 & 0.550389 & 0.070^{*} \\ \text { C38 } & 0.7565(2) & 0.3953(2) & 0.54571(15) & 0.0600(7) \\ \text { H38 } & 0.717158 & 0.457127 & 0.560291 & 0.072^{*} \\ \text { C39 } & 0.6895(2) & 0.3055(2) & 0.53040(13) & 0.0454(5) \\ \text { H39 } & 0.604491 & 0.306589 & 0.535436 & 0.055^{*} \\ \text { C40 } & -0.11856(18) & 0.76483(18) & 0.08531(10) & 0.0351(4) \\ \text { C41 } & -0.2240(2) & 0.7879(2) & 0.12733(11) & 0.0417(5) \\ \text { C42 } & -0.3247(2) & 0.7133(3) & 0.13934(14) & 0.0596(7) \\ \text { C43 } & -0.4237(3) & 0.7394(4) & 0.17739(19) & 0.0996(12) \\ \text { H43 } & -0.491888 & 0.690437 & 0.184663 & 0.119^{*} \\ \text { C44 } & -0.4210(4) & 0.8365(5) & 0.2040(2) & 0.1126(15) \\ \text { H44 } & -0.487079 & 0.852323 & 0.230315 & 0.135^{*} \\ \text { C45 } & -0.3231(4) & 0.9112(4) & 0.19291(19) & 0.1003(12) \\ \text { H45 } & -0.322902 & 0.977912 & 0.210964 & 0.120^{*} \\ \text { C46 } & -0.2246(3) & 0.8869(3) & 0.15467(14) & 0.0654(7) \\ \text { H46 } & -0.157687 & 0.937517 & 0.147131 & 0.079^{*} \\ \text { C47 } & 0.03259(19) & 0.81423(19) & -0.13648(11) & 0.0389(5) \\ \text { C48 } & -0.01849(19) & 0.90816(18) & -0.19807(10) & 0.0369(5) \\ \text { C49 } & 0.0107(2) & 0.9058(2) & -0.26616(11) & 0.0408(5) \\ \text { C50 } & -0.0378(2) & 0.9945(2) & -0.32279(12) & 0.0532(6) \\ \text { H50 } & -0.018213 & 0.992567 & -0.368002 & 0.064^{*} \\ \text { C51 } & -0.1141(3) & 1.0846(2) & -0.31211(13) & 0.0557(6) \\ \text { H51 } & -0.146484 & 1.143341 & -0.350112 & 0.067^{*} \\ \text { C52 } & -0.1430(3) & 1.0884(2) & -0.24526(13) & 0.0585(7) \\ \text { H52 } & -0.194411 & 1.150024 & -0.238205 & 0.070^{*} \\ \text { C53 } & -0.0959(2) & 1.0013(2) & -0.18913(12) & 0.0492(6) \\ \text { H53 } & -0.116154 & 1.004531 & -0.144223 & 0.059^{*} \\ & & & & \end{array}$

Atomic displacement parameters $\left(\AA^{2}\right)$

\begin{tabular}{lllllll}
\hline & $U^{11}$ & $U^{22}$ & $U^{33}$ & $U^{12}$ & $U^{13}$ & $U^{23}$ \\
\hline Co1 & $0.02255(13)$ & $0.02972(14)$ & $0.03064(14)$ & $-0.00244(10)$ & $-0.00050(10)$ & $-0.01000(11)$ \\
Co2 & $0.03023(15)$ & $0.03340(15)$ & $0.03044(14)$ & $-0.00662(11)$ & $-0.00272(11)$ & $-0.00606(11)$ \\
O1 & $0.0320(8)$ & $0.0574(10)$ & $0.0669(10)$ & $-0.0087(7)$ & $0.0140(7)$ & $-0.0352(8)$ \\
O2 & $0.0345(8)$ & $0.0414(8)$ & $0.0535(9)$ & $-0.0060(6)$ & $0.0036(7)$ & $-0.0236(7)$ \\
O3 & $0.0613(13)$ & $0.0969(17)$ & $0.171(2)$ & $-0.0421(12)$ & $0.0432(14)$ & $-0.0927(18)$ \\
O4 & $0.0227(7)$ & $0.0500(9)$ & $0.0494(9)$ & $-0.0074(6)$ & $0.0033(6)$ & $-0.0153(7)$ \\
O5 & $0.0289(7)$ & $0.0325(7)$ & $0.0456(8)$ & $-0.0027(6)$ & $-0.0020(6)$ & $-0.0167(6)$ \\
O6 & $0.0283(8)$ & $0.0663(11)$ & $0.1028(15)$ & $-0.0096(8)$ & $0.0154(9)$ & $-0.0514(11)$ \\
O7 & $0.0401(8)$ & $0.0434(8)$ & $0.0424(8)$ & $-0.0058(6)$ & $-0.0009(6)$ & $-0.0182(7)$ \\
O8 & $0.0373(8)$ & $0.0409(8)$ & $0.0534(9)$ & $-0.0087(7)$ & $0.0015(7)$ & $-0.0127(7)$
\end{tabular}




\begin{tabular}{|c|c|c|c|c|c|c|}
\hline O9 & $0.0702(15)$ & $0.1036(17)$ & $0.1071(18)$ & $-0.0508(13)$ & $0.0321(13)$ & $-0.0558(15)$ \\
\hline $\mathrm{O} 10$ & $0.0473(9)$ & $0.0540(9)$ & $0.0305(8)$ & $-0.0016(7)$ & $-0.0051(7)$ & $-0.0036(7)$ \\
\hline $\mathrm{O} 11$ & $0.0608(11)$ & $0.0557(10)$ & $0.0417(9)$ & $0.0114(8)$ & $-0.0053(8)$ & $-0.0064(8)$ \\
\hline $\mathrm{O} 12$ & $0.0549(11)$ & $0.0758(13)$ & $0.0468(10)$ & $0.0011(9)$ & $0.0071(9)$ & $-0.0164(10)$ \\
\hline $\mathrm{O} 13$ & $0.0557(10)$ & $0.0367(8)$ & $0.0393(9)$ & $0.0024(7)$ & $-0.0089(8)$ & $-0.0092(7)$ \\
\hline N1 & $0.0301(9)$ & $0.0372(9)$ & $0.0343(9)$ & $-0.0013(7)$ & $-0.0024(7)$ & $-0.0094(7)$ \\
\hline N2 & $0.0479(11)$ & $0.0517(12)$ & $0.0379(10)$ & $0.0049(9)$ & $-0.0037(9)$ & $-0.0049(9)$ \\
\hline N3 & $0.0268(8)$ & $0.0334(9)$ & $0.0318(8)$ & $-0.0035(7)$ & $-0.0002(7)$ & $-0.0064(7)$ \\
\hline N4 & $0.0327(9)$ & $0.0344(9)$ & $0.0316(8)$ & $-0.0037(7)$ & $-0.0008(7)$ & $-0.0070(7)$ \\
\hline N5 & $0.0326(9)$ & $0.0345(9)$ & $0.0385(9)$ & $-0.0076(7)$ & $0.0018(7)$ & $-0.0068(7)$ \\
\hline $\mathrm{C} 1$ & $0.0350(11)$ & $0.0470(13)$ & $0.0396(11)$ & $-0.0116(9)$ & $-0.0019(9)$ & $-0.0040(10)$ \\
\hline $\mathrm{C} 2$ & $0.0302(11)$ & $0.0512(13)$ & $0.0409(12)$ & $-0.0069(9)$ & $-0.0067(9)$ & $-0.0073(10)$ \\
\hline $\mathrm{C} 3$ & $0.0331(10)$ & $0.0342(10)$ & $0.0336(10)$ & $-0.0006(8)$ & $-0.0004(8)$ & $-0.0118(8)$ \\
\hline $\mathrm{C} 4$ & $0.0303(11)$ & $0.0445(12)$ & $0.0394(11)$ & $-0.0076(9)$ & $0.0015(9)$ & $-0.0044(9)$ \\
\hline $\mathrm{C} 5$ & $0.0275(10)$ & $0.0487(13)$ & $0.0388(11)$ & $-0.0029(9)$ & $-0.0024(9)$ & $-0.0067(10)$ \\
\hline C6 & $0.0419(12)$ & $0.0562(14)$ & $0.0381(12)$ & $0.0028(10)$ & $-0.0074(10)$ & $-0.0149(11)$ \\
\hline $\mathrm{C} 7$ & $0.0382(11)$ & $0.0393(11)$ & $0.0386(11)$ & $-0.0034(9)$ & $-0.0020(9)$ & $-0.0108(9)$ \\
\hline $\mathrm{C} 8$ & $0.0309(10)$ & $0.0381(11)$ & $0.0345(10)$ & $0.0023(8)$ & $0.0007(8)$ & $-0.0115(9)$ \\
\hline C9 & $0.0421(12)$ & $0.0433(12)$ & $0.0436(12)$ & $-0.0082(10)$ & $-0.0071(10)$ & $-0.0037(10)$ \\
\hline $\mathrm{C} 10$ & $0.0566(15)$ & $0.0435(13)$ & $0.0474(13)$ & $-0.0068(11)$ & $-0.0021(11)$ & $-0.0002(11)$ \\
\hline $\mathrm{C} 11$ & $0.0298(10)$ & $0.0392(11)$ & $0.0411(11)$ & $-0.0077(8)$ & $-0.0068(9)$ & $-0.0072(9)$ \\
\hline $\mathrm{C} 12$ & $0.0310(10)$ & $0.0360(11)$ & $0.0396(11)$ & $-0.0102(8)$ & $-0.0012(9)$ & $-0.0034(9)$ \\
\hline $\mathrm{C} 13$ & $0.0275(10)$ & $0.0308(10)$ & $0.0324(10)$ & $-0.0008(8)$ & $0.0002(8)$ & $-0.0099(8)$ \\
\hline $\mathrm{C} 14$ & $0.0230(9)$ & $0.0440(12)$ & $0.0393(11)$ & $-0.0046(8)$ & $-0.0035(8)$ & $-0.0055(9)$ \\
\hline $\mathrm{C} 15$ & $0.0248(10)$ & $0.0434(12)$ & $0.0369(11)$ & $-0.0058(8)$ & $0.0024(8)$ & $-0.0023(9)$ \\
\hline $\mathrm{C} 16$ & $0.0434(12)$ & $0.0323(11)$ & $0.0352(11)$ & $-0.0083(9)$ & $-0.0019(9)$ & $-0.0053(9)$ \\
\hline $\mathrm{C} 17$ & $0.0386(11)$ & $0.0370(11)$ & $0.0366(11)$ & $-0.0077(9)$ & $-0.0060(9)$ & $-0.0105(9)$ \\
\hline C18 & $0.0257(9)$ & $0.0331(10)$ & $0.0312(10)$ & $-0.0001(8)$ & $0.0031(8)$ & $-0.0083(8)$ \\
\hline C19 & $0.0350(11)$ & $0.0317(10)$ & $0.0358(10)$ & $-0.0074(8)$ & $0.0002(8)$ & $-0.0070(8)$ \\
\hline $\mathrm{C} 20$ & $0.0355(11)$ & $0.0389(11)$ & $0.0339(10)$ & $-0.0087(9)$ & $-0.0038(8)$ & $-0.0092(9)$ \\
\hline $\mathrm{C} 21$ & $0.0431(14)$ & $0.0479(14)$ & $0.106(2)$ & $-0.0135(11)$ & $0.0171(14)$ & $-0.0420(15)$ \\
\hline $\mathrm{C} 22$ & $0.0359(13)$ & $0.0581(16)$ & $0.122(2)$ & $-0.0137(11)$ & $0.0255(14)$ & $-0.0501(17)$ \\
\hline $\mathrm{C} 23$ & $0.0341(11)$ & $0.0350(11)$ & $0.0393(11)$ & $-0.0083(8)$ & $0.0037(9)$ & $-0.0094(9)$ \\
\hline $\mathrm{C} 24$ & $0.0360(11)$ & $0.0338(11)$ & $0.0580(14)$ & $-0.0054(9)$ & $0.0032(10)$ & $-0.0158(10)$ \\
\hline $\mathrm{C} 25$ & $0.0325(11)$ & $0.0388(12)$ & $0.0526(13)$ & $-0.0029(9)$ & $0.0028(10)$ & $-0.0109(10)$ \\
\hline $\mathrm{C} 26$ & $0.0299(10)$ & $0.0381(11)$ & $0.0357(10)$ & $-0.0003(8)$ & $0.0004(8)$ & $-0.0121(9)$ \\
\hline $\mathrm{C} 27$ & $0.0330(11)$ & $0.0404(12)$ & $0.0462(12)$ & $-0.0006(9)$ & $0.0023(9)$ & $-0.0201(10)$ \\
\hline $\mathrm{C} 28$ & $0.0446(14)$ & $0.0581(15)$ & $0.0853(19)$ & $-0.0104(11)$ & $0.0096(13)$ & $-0.0407(14)$ \\
\hline $\mathrm{C} 29$ & $0.078(2)$ & $0.073(2)$ & $0.129(3)$ & $-0.0116(16)$ & $0.013(2)$ & $-0.071(2)$ \\
\hline $\mathrm{C} 30$ & $0.078(2)$ & $0.091(2)$ & $0.138(3)$ & $0.0048(18)$ & $0.023(2)$ & $-0.081(2)$ \\
\hline $\mathrm{C} 31$ & $0.0585(18)$ & $0.097(2)$ & $0.128(3)$ & $-0.0012(17)$ & $0.0335(19)$ & $-0.070(2)$ \\
\hline $\mathrm{C} 32$ & $0.0396(13)$ & $0.0647(16)$ & $0.0810(19)$ & $-0.0055(12)$ & $0.0138(13)$ & $-0.0363(15)$ \\
\hline $\mathrm{C} 33$ & $0.0254(10)$ & $0.0308(10)$ & $0.0246(9)$ & $-0.0042(7)$ & $-0.0008(7)$ & $-0.0029(7)$ \\
\hline $\mathrm{C} 34$ & $0.0272(9)$ & $0.0293(10)$ & $0.0302(10)$ & $-0.0034(7)$ & $0.0011(8)$ & $-0.0079(8)$ \\
\hline $\mathrm{C} 35$ & $0.0286(10)$ & $0.0380(11)$ & $0.0457(12)$ & $-0.0073(8)$ & $0.0062(9)$ & $-0.0139(9)$ \\
\hline $\mathrm{C} 36$ & $0.0348(12)$ & $0.0587(15)$ & $0.0755(17)$ & $-0.0201(11)$ & $0.0094(12)$ & $-0.0292(13)$ \\
\hline $\mathrm{C} 37$ & $0.0599(16)$ & $0.0484(14)$ & $0.0749(18)$ & $-0.0248(12)$ & $0.0078(13)$ & $-0.0301(13)$ \\
\hline C38 & $0.0595(16)$ & $0.0454(14)$ & $0.087(2)$ & $-0.0055(12)$ & $0.0106(14)$ & $-0.0386(14)$ \\
\hline
\end{tabular}




\begin{tabular}{lllllll} 
& & & & & \\
C39 & $0.0342(11)$ & $0.0426(12)$ & $0.0652(15)$ & $-0.0023(9)$ & $0.0045(10)$ & $-0.0242(11)$ \\
C40 & $0.0346(11)$ & $0.0376(11)$ & $0.0318(10)$ & $-0.0014(9)$ & $-0.0060(8)$ & $-0.0071(9)$ \\
C41 & $0.0383(12)$ & $0.0491(13)$ & $0.0380(11)$ & $0.0010(10)$ & $-0.0012(9)$ & $-0.0125(10)$ \\
C42 & $0.0449(14)$ & $0.084(2)$ & $0.0532(15)$ & $-0.0119(13)$ & $0.0088(12)$ & $-0.0232(14)$ \\
C43 & $0.0530(19)$ & $0.157(4)$ & $0.094(3)$ & $-0.018(2)$ & $0.0313(18)$ & $-0.042(3)$ \\
C44 & $0.087(3)$ & $0.166(4)$ & $0.095(3)$ & $0.029(3)$ & $0.030(2)$ & $-0.056(3)$ \\
C45 & $0.116(3)$ & $0.112(3)$ & $0.089(3)$ & $0.033(3)$ & $0.010(2)$ & $-0.058(2)$ \\
C46 & $0.0744(19)$ & $0.0665(18)$ & $0.0634(17)$ & $0.0101(14)$ & $-0.0003(14)$ & $-0.0325(14)$ \\
C47 & $0.0361(11)$ & $0.0408(12)$ & $0.0378(12)$ & $-0.0121(9)$ & $-0.0052(9)$ & $-0.0061(9)$ \\
C48 & $0.0381(11)$ & $0.0371(11)$ & $0.0341(10)$ & $-0.0133(9)$ & $-0.0041(9)$ & $-0.0057(9)$ \\
C49 & $0.0376(11)$ & $0.0456(12)$ & $0.0382(11)$ & $-0.0123(9)$ & $0.0011(9)$ & $-0.0088(10)$ \\
C50 & $0.0615(16)$ & $0.0614(16)$ & $0.0324(12)$ & $-0.0167(13)$ & $-0.0008(11)$ & $-0.0041(11)$ \\
C51 & $0.0699(17)$ & $0.0436(14)$ & $0.0453(14)$ & $-0.0119(12)$ & $-0.0150(12)$ & $0.0034(11)$ \\
C52 & $0.0750(18)$ & $0.0418(13)$ & $0.0565(15)$ & $0.0047(12)$ & $-0.0135(13)$ & $-0.0100(12)$ \\
C53 & $0.0622(15)$ & $0.0460(13)$ & $0.0398(12)$ & $-0.0014(11)$ & $-0.0079(11)$ & $-0.0124(10)$ \\
& & & & & & \\
\hline
\end{tabular}

Geometric parameters $\left(A,{ }^{\circ}\right)$

\begin{tabular}{llll}
\hline $\mathrm{Co} 1-\mathrm{O} 1$ & $2.1772(15)$ & $\mathrm{C} 14-\mathrm{H} 14$ & 0.9300 \\
$\mathrm{C} 1-\mathrm{O} 2$ & $2.1769(14)$ & $\mathrm{C} 14-\mathrm{C} 15$ & $1.377(3)$ \\
$\mathrm{Co} 1-\mathrm{O} 4$ & $2.0408(14)$ & $\mathrm{C} 15-\mathrm{H} 15$ & 0.9300 \\
$\mathrm{C} 1-\mathrm{O} 5 \mathrm{i}$ & $2.0686(13)$ & $\mathrm{C} 16-\mathrm{H} 16$ & 0.9300 \\
$\mathrm{Co} 1-\mathrm{N} 1$ & $2.1412(16)$ & $\mathrm{C} 16-\mathrm{C} 17$ & $1.376(3)$ \\
$\mathrm{Co} 1-\mathrm{N} 3$ & $2.1421(16)$ & $\mathrm{C} 17-\mathrm{H} 17$ & 0.9300 \\
$\mathrm{C} 2-\mathrm{O} 7$ & $2.2348(15)$ & $\mathrm{C} 17-\mathrm{C} 18$ & $1.393(3)$ \\
$\mathrm{Co} 2-\mathrm{O} 8$ & $2.1135(15)$ & $\mathrm{C} 18-\mathrm{C} 19$ & $1.389(3)$ \\
$\mathrm{C} 2-\mathrm{O} 10$ & $2.0739(14)$ & $\mathrm{C} 19-\mathrm{H} 19$ & 0.9300 \\
$\mathrm{C} 2-\mathrm{O} 13$ & $2.0717(16)$ & $\mathrm{C} 19-\mathrm{C} 20$ & $1.375(3)$ \\
$\mathrm{C} 2-\mathrm{N} 4$ & $2.1568(16)$ & $\mathrm{C} 20-\mathrm{H} 20$ & 0.9300 \\
$\mathrm{C} 2-\mathrm{N} 5$ & $2.1177(16)$ & $\mathrm{C} 21-\mathrm{H} 21$ & 0.9300 \\
$\mathrm{Co} 2-\mathrm{C} 40$ & $2.514(2)$ & $\mathrm{C} 21-\mathrm{C} 22$ & $1.375(3)$ \\
$\mathrm{O} 1-\mathrm{C} 26$ & $1.270(2)$ & $\mathrm{C} 22-\mathrm{H} 22$ & 0.9300 \\
$\mathrm{O} 2-\mathrm{C} 26$ & $1.247(2)$ & $\mathrm{C} 22-\mathrm{C} 23$ & $1.379(3)$ \\
$\mathrm{O} 3-\mathrm{H} 3$ & $0.845(10)$ & $\mathrm{C} 23-\mathrm{C} 23^{\mathrm{ii}}$ & $1.488(4)$ \\
$\mathrm{O} 3-\mathrm{C} 28$ & $1.346(3)$ & $\mathrm{C} 23-\mathrm{C} 24$ & $1.379(3)$ \\
$\mathrm{O} 4-\mathrm{C} 33$ & $1.244(2)$ & $\mathrm{C} 24-\mathrm{H} 24$ & 0.9300 \\
$\mathrm{O} 5-\mathrm{C} 33$ & $1.275(2)$ & $\mathrm{C} 24-\mathrm{C} 25$ & $1.378(3)$ \\
$\mathrm{O} 6-\mathrm{H} 8$ & $0.838(10)$ & $\mathrm{C} 25-\mathrm{H} 25$ & 0.9300 \\
$\mathrm{O} 6-\mathrm{C} 35$ & $1.348(3)$ & $\mathrm{C} 26-\mathrm{C} 27$ & $1.484(3)$ \\
$\mathrm{O} 7-\mathrm{C} 40$ & $1.272(2)$ & $\mathrm{C} 27-\mathrm{C} 28$ & $1.391(3)$ \\
$\mathrm{O} 8-\mathrm{C} 40$ & $1.254(2)$ & $\mathrm{C} 27-\mathrm{C} 32$ & $1.380(3)$ \\
$\mathrm{O} 9-\mathrm{H} 13$ & $0.839(10)$ & $\mathrm{C} 28-\mathrm{C} 29$ & $1.386(4)$ \\
$\mathrm{O} 9-\mathrm{C} 42$ & $1.346(3)$ & $\mathrm{C} 29-\mathrm{H} 29$ & 0.9300 \\
$\mathrm{O} 10-\mathrm{C} 47$ & $1.261(3)$ & $\mathrm{C} 29-\mathrm{C} 30$ & $1.365(4)$ \\
$\mathrm{O} 11-\mathrm{C} 47$ & $1.266(3)$ & $\mathrm{C} 30-\mathrm{H} 30$ & 0.9300 \\
$\mathrm{O} 12-\mathrm{H} 18$ & $0.853(10)$ & $\mathrm{C} 30-\mathrm{C} 31$ & $1.366(4)$ \\
$\mathrm{O} 12-\mathrm{C} 49$ & $1.348(3)$ & $\mathrm{C} 31-\mathrm{H} 31$ & $1.379(4)$ \\
$\mathrm{O} 13-\mathrm{H} 26$ & $0.837(10)$ & $\mathrm{C} 31-\mathrm{C} 32$ &
\end{tabular}




\begin{tabular}{|c|c|c|c|}
\hline $\mathrm{O} 13-\mathrm{H} 27$ & $0.832(10)$ & $\mathrm{C} 32-\mathrm{H} 32$ & 0.9300 \\
\hline $\mathrm{N} 1-\mathrm{C} 1$ & $1.341(3)$ & $\mathrm{C} 33-\mathrm{C} 34$ & $1.486(3)$ \\
\hline $\mathrm{N} 1-\mathrm{C} 5$ & $1.338(3)$ & $\mathrm{C} 34-\mathrm{C} 35$ & $1.398(3)$ \\
\hline $\mathrm{N} 2-\mathrm{C} 6$ & $1.338(3)$ & C34-C39 & $1.390(3)$ \\
\hline $\mathrm{N} 2-\mathrm{C} 10$ & $1.333(3)$ & $\mathrm{C} 35-\mathrm{C} 36$ & $1.392(3)$ \\
\hline N3-C11 & $1.340(2)$ & $\mathrm{C} 36-\mathrm{H} 36$ & 0.9300 \\
\hline $\mathrm{N} 3-\mathrm{C} 15$ & $1.339(2)$ & $\mathrm{C} 36-\mathrm{C} 37$ & $1.369(3)$ \\
\hline $\mathrm{N} 4-\mathrm{C} 16$ & $1.338(3)$ & C37-H37 & 0.9300 \\
\hline $\mathrm{N} 4-\mathrm{C} 20$ & $1.338(2)$ & $\mathrm{C} 37-\mathrm{C} 38$ & $1.373(4)$ \\
\hline $\mathrm{N} 5-\mathrm{C} 21$ & $1.329(3)$ & C38-H38 & 0.9300 \\
\hline N5-C25 & $1.330(3)$ & $\mathrm{C} 38-\mathrm{C} 39$ & $1.378(3)$ \\
\hline $\mathrm{C} 1-\mathrm{H} 1$ & 0.9300 & C39-H39 & 0.9300 \\
\hline $\mathrm{C} 1-\mathrm{C} 2$ & $1.370(3)$ & $\mathrm{C} 40-\mathrm{C} 41$ & $1.478(3)$ \\
\hline $\mathrm{C} 2-\mathrm{H} 2$ & 0.9300 & $\mathrm{C} 41-\mathrm{C} 42$ & $1.388(3)$ \\
\hline $\mathrm{C} 2-\mathrm{C} 3$ & $1.387(3)$ & $\mathrm{C} 41-\mathrm{C} 46$ & $1.386(3)$ \\
\hline $\mathrm{C} 3-\mathrm{C} 4$ & $1.391(3)$ & $\mathrm{C} 42-\mathrm{C} 43$ & $1.387(4)$ \\
\hline $\mathrm{C} 3-\mathrm{C} 8$ & $1.479(3)$ & $\mathrm{C} 43-\mathrm{H} 43$ & 0.9300 \\
\hline $\mathrm{C} 4-\mathrm{H} 4$ & 0.9300 & $\mathrm{C} 43-\mathrm{C} 44$ & $1.358(6)$ \\
\hline $\mathrm{C} 4-\mathrm{C} 5$ & $1.375(3)$ & $\mathrm{C} 44-\mathrm{H} 44$ & 0.9300 \\
\hline $\mathrm{C} 5-\mathrm{H} 5$ & 0.9300 & $\mathrm{C} 44-\mathrm{C} 45$ & $1.364(6)$ \\
\hline $\mathrm{C} 6-\mathrm{H} 6$ & 0.9300 & $\mathrm{C} 45-\mathrm{H} 45$ & 0.9300 \\
\hline $\mathrm{C} 6-\mathrm{C} 7$ & $1.373(3)$ & $\mathrm{C} 45-\mathrm{C} 46$ & $1.379(4)$ \\
\hline $\mathrm{C} 7-\mathrm{H} 7$ & 0.9300 & $\mathrm{C} 46-\mathrm{H} 46$ & 0.9300 \\
\hline $\mathrm{C} 7-\mathrm{C} 8$ & $1.392(3)$ & $\mathrm{C} 47-\mathrm{C} 48$ & $1.494(3)$ \\
\hline $\mathrm{C} 8-\mathrm{C} 9$ & 1.385 & $\mathrm{C} 48-\mathrm{C} 49$ & $1.398(3)$ \\
\hline $\mathrm{C} 9-\mathrm{H} 9 \mathrm{~A}$ & 0.9300 & $\mathrm{C} 48-\mathrm{C} 53$ & $1.392(3)$ \\
\hline $\mathrm{C} 9-\mathrm{C} 10$ & $1.377(3)$ & $\mathrm{C} 49-\mathrm{C} 50$ & $1.393(3)$ \\
\hline $\mathrm{C} 10-\mathrm{H} 10$ & 0.9300 & $\mathrm{C} 50-\mathrm{H} 50$ & 0.9300 \\
\hline C11-H11 & 0.9300 & $\mathrm{C} 50-\mathrm{C} 51$ & $1.369(4)$ \\
\hline $\mathrm{C} 11-\mathrm{C} 12$ & $1.372(3)$ & $\mathrm{C} 51-\mathrm{H} 51$ & 0.9300 \\
\hline $\mathrm{C} 12-\mathrm{H} 12$ & 0.9300 & $\mathrm{C} 51-\mathrm{C} 52$ & $1.378(4)$ \\
\hline $\mathrm{C} 12-\mathrm{C} 13$ & $1.390(3)$ & $\mathrm{C} 52-\mathrm{H} 52$ & 0.9300 \\
\hline $\mathrm{C} 13-\mathrm{C} 14$ & $1.391(3)$ & $\mathrm{C} 52-\mathrm{C} 53$ & $1.372(3)$ \\
\hline $\mathrm{C} 13-\mathrm{C} 18$ & $1.474(3)$ & $\mathrm{C} 53-\mathrm{H} 53$ & 0.9300 \\
\hline $\mathrm{O} 2-\mathrm{Co} 1-\mathrm{O} 1$ & $59.82(5)$ & $\mathrm{C} 18-\mathrm{C} 17-\mathrm{H} 17$ & 120.1 \\
\hline $\mathrm{O} 4-\mathrm{Co} 1-\mathrm{O} 1$ & $91.69(6)$ & $\mathrm{C} 17-\mathrm{C} 18-\mathrm{C} 13$ & $121.87(17)$ \\
\hline $\mathrm{O} 4-\mathrm{Co} 1-\mathrm{O} 2$ & $151.47(6)$ & $\mathrm{C} 19-\mathrm{C} 18-\mathrm{C} 13$ & $121.51(17)$ \\
\hline $\mathrm{O} 4-\mathrm{Co} 1-\mathrm{O} 5^{\mathrm{i}}$ & $117.97(6)$ & $\mathrm{C} 19-\mathrm{C} 18-\mathrm{C} 17$ & $116.62(17)$ \\
\hline $\mathrm{O} 4-\mathrm{Co} 1-\mathrm{N} 1$ & $91.32(6)$ & C18-C19-H19 & 120.0 \\
\hline $\mathrm{O} 4-\mathrm{Co} 1-\mathrm{N} 3$ & $87.16(6)$ & $\mathrm{C} 20-\mathrm{C} 19-\mathrm{C} 18$ & $119.94(18)$ \\
\hline $\mathrm{O} 5-\mathrm{Co} 1-\mathrm{O} 1$ & $150.29(5)$ & $\mathrm{C} 20-\mathrm{C} 19-\mathrm{H} 19$ & 120.0 \\
\hline $\mathrm{O}^{\mathrm{i}}-\mathrm{Co} 1-\mathrm{O} 2$ & $90.48(5)$ & $\mathrm{N} 4-\mathrm{C} 20-\mathrm{C} 19$ & $123.42(18)$ \\
\hline $\mathrm{O} 5^{\mathrm{i}}-\mathrm{Co} 1-\mathrm{N} 1$ & $89.68(6)$ & $\mathrm{N} 4-\mathrm{C} 20-\mathrm{H} 20$ & 118.3 \\
\hline $\mathrm{O}^{\mathrm{i}}-\mathrm{Co} 1-\mathrm{N} 3$ & $89.31(6)$ & $\mathrm{C} 19-\mathrm{C} 20-\mathrm{H} 20$ & 118.3 \\
\hline $\mathrm{N} 1-\mathrm{Co} 1-\mathrm{O} 1$ & $91.38(6)$ & $\mathrm{N} 5-\mathrm{C} 21-\mathrm{H} 21$ & 118.3 \\
\hline $\mathrm{N} 1-\mathrm{Co} 1-\mathrm{O} 2$ & $90.90(6)$ & $\mathrm{N} 5-\mathrm{C} 21-\mathrm{C} 22$ & $123.4(2)$ \\
\hline $\mathrm{N} 1-\mathrm{Co} 1-\mathrm{N} 3$ & $177.53(6)$ & $\mathrm{C} 22-\mathrm{C} 21-\mathrm{H} 21$ & 118.3 \\
\hline
\end{tabular}




\begin{tabular}{|c|c|c|c|}
\hline $\mathrm{N} 3-\mathrm{Co} 1-\mathrm{O} 1$ & $90.61(6)$ & $\mathrm{C} 21-\mathrm{C} 22-\mathrm{H} 22$ & 119.8 \\
\hline $\mathrm{N} 3-\mathrm{Co} 1-\mathrm{O} 2$ & $91.37(6)$ & $\mathrm{C} 21-\mathrm{C} 22-\mathrm{C} 23$ & $120.4(2)$ \\
\hline $\mathrm{O} 7-\mathrm{Co} 2-\mathrm{C} 40$ & $30.36(6)$ & $\mathrm{C} 23-\mathrm{C} 22-\mathrm{H} 22$ & 119.8 \\
\hline $\mathrm{O} 8-\mathrm{Co} 2-\mathrm{O} 7$ & $60.20(5)$ & $\mathrm{C} 22-\mathrm{C} 23-\mathrm{C} 23^{\mathrm{ii}}$ & $122.0(2)$ \\
\hline $\mathrm{O} 8-\mathrm{Co} 2-\mathrm{N} 4$ & $89.11(6)$ & $\mathrm{C} 22-\mathrm{C} 23-\mathrm{C} 24$ & $115.94(19)$ \\
\hline $\mathrm{O} 8-\mathrm{Co} 2-\mathrm{N} 5$ & $95.55(6)$ & $\mathrm{C} 24-\mathrm{C} 23-\mathrm{C} 23^{\mathrm{ii}}$ & $122.1(2)$ \\
\hline $\mathrm{O} 8-\mathrm{Co} 2-\mathrm{C} 40$ & $29.87(6)$ & $\mathrm{C} 23-\mathrm{C} 24-\mathrm{H} 24$ & 119.7 \\
\hline $\mathrm{O} 10-\mathrm{Co} 2-\mathrm{O} 7$ & $91.19(6)$ & $\mathrm{C} 25-\mathrm{C} 24-\mathrm{C} 23$ & $120.6(2)$ \\
\hline $\mathrm{O} 10-\mathrm{Co} 2-\mathrm{O} 8$ & $93.29(6)$ & $\mathrm{C} 25-\mathrm{C} 24-\mathrm{H} 24$ & 119.7 \\
\hline $\mathrm{O} 10-\mathrm{Co} 2-\mathrm{N} 4$ & $173.40(6)$ & $\mathrm{N} 5-\mathrm{C} 25-\mathrm{C} 24$ & $122.9(2)$ \\
\hline $\mathrm{O} 10-\mathrm{Co} 2-\mathrm{N} 5$ & $93.17(6)$ & $\mathrm{N} 5-\mathrm{C} 25-\mathrm{H} 25$ & 118.5 \\
\hline $\mathrm{O} 10-\mathrm{Co} 2-\mathrm{C} 40$ & $93.68(6)$ & $\mathrm{C} 24-\mathrm{C} 25-\mathrm{H} 25$ & 118.5 \\
\hline $\mathrm{O} 13-\mathrm{Co} 2-\mathrm{O} 7$ & $107.21(6)$ & $\mathrm{O} 1-\mathrm{C} 26-\mathrm{C} 27$ & $118.92(18)$ \\
\hline $\mathrm{O} 13-\mathrm{Co} 2-\mathrm{O} 8$ & $167.09(6)$ & $\mathrm{O} 2-\mathrm{C} 26-\mathrm{O} 1$ & $119.20(18)$ \\
\hline $\mathrm{O} 13-\mathrm{Co} 2-\mathrm{O} 10$ & $89.66(6)$ & $\mathrm{O} 2-\mathrm{C} 26-\mathrm{C} 27$ & $121.87(18)$ \\
\hline $\mathrm{O} 13-\mathrm{Co} 2-\mathrm{N} 4$ & $86.68(6)$ & $\mathrm{C} 28-\mathrm{C} 27-\mathrm{C} 26$ & $120.90(19)$ \\
\hline $\mathrm{O} 13-\mathrm{Co} 2-\mathrm{N} 5$ & $96.83(7)$ & $\mathrm{C} 32-\mathrm{C} 27-\mathrm{C} 26$ & $120.2(2)$ \\
\hline $\mathrm{O} 13-\mathrm{Co} 2-\mathrm{C} 40$ & $137.40(7)$ & $\mathrm{C} 32-\mathrm{C} 27-\mathrm{C} 28$ & $118.9(2)$ \\
\hline $\mathrm{N} 4-\mathrm{Co} 2-\mathrm{O} 7$ & $84.66(6)$ & $\mathrm{O} 3-\mathrm{C} 28-\mathrm{C} 27$ & $122.1(2)$ \\
\hline $\mathrm{N} 4-\mathrm{Co} 2-\mathrm{C} 40$ & $85.34(6)$ & $\mathrm{O} 3-\mathrm{C} 28-\mathrm{C} 29$ & $118.0(2)$ \\
\hline $\mathrm{N} 5-\mathrm{Co} 2-\mathrm{O} 7$ & $155.58(6)$ & $\mathrm{C} 29-\mathrm{C} 28-\mathrm{C} 27$ & $119.8(2)$ \\
\hline $\mathrm{N} 5-\mathrm{Co} 2-\mathrm{N} 4$ & $92.71(6)$ & $\mathrm{C} 28-\mathrm{C} 29-\mathrm{H} 29$ & 120.0 \\
\hline $\mathrm{N} 5-\mathrm{Co} 2-\mathrm{C} 40$ & $125.28(7)$ & $\mathrm{C} 30-\mathrm{C} 29-\mathrm{C} 28$ & $119.9(3)$ \\
\hline $\mathrm{C} 26-\mathrm{O} 1-\mathrm{Co} 1$ & $90.17(12)$ & $\mathrm{C} 30-\mathrm{C} 29-\mathrm{H} 29$ & 120.0 \\
\hline $\mathrm{C} 26-\mathrm{O} 2-\mathrm{Co} 1$ & $90.79(12)$ & $\mathrm{C} 29-\mathrm{C} 30-\mathrm{H} 30$ & 119.6 \\
\hline $\mathrm{C} 28-\mathrm{O} 3-\mathrm{H} 3$ & $105(3)$ & $\mathrm{C} 29-\mathrm{C} 30-\mathrm{C} 31$ & $120.9(3)$ \\
\hline $\mathrm{C} 33-\mathrm{O} 4-\mathrm{Co} 1$ & $165.49(14)$ & $\mathrm{C} 31-\mathrm{C} 30-\mathrm{H} 30$ & 119.6 \\
\hline $\mathrm{C} 33-\mathrm{O} 5-\mathrm{Co}^{\mathrm{i}}$ & $117.07(11)$ & $\mathrm{C} 30-\mathrm{C} 31-\mathrm{H} 31$ & 120.2 \\
\hline $\mathrm{C} 35-\mathrm{O} 6-\mathrm{H} 8$ & $110(2)$ & $\mathrm{C} 30-\mathrm{C} 31-\mathrm{C} 32$ & $119.6(3)$ \\
\hline $\mathrm{C} 40-\mathrm{O} 7-\mathrm{Co} 2$ & $87.04(12)$ & $\mathrm{C} 32-\mathrm{C} 31-\mathrm{H} 31$ & 120.2 \\
\hline $\mathrm{C} 40-\mathrm{O} 8-\mathrm{Co} 2$ & $93.04(12)$ & $\mathrm{C} 27-\mathrm{C} 32-\mathrm{H} 32$ & 119.6 \\
\hline $\mathrm{C} 42-\mathrm{O} 9-\mathrm{H} 13$ & $104(3)$ & $\mathrm{C} 31-\mathrm{C} 32-\mathrm{C} 27$ & $120.7(2)$ \\
\hline $\mathrm{C} 47-\mathrm{O} 10-\mathrm{Co} 2$ & $129.12(15)$ & C31-C32-H32 & 119.6 \\
\hline $\mathrm{C} 49-\mathrm{O} 12-\mathrm{H} 18$ & $105(2)$ & $\mathrm{O} 4-\mathrm{C} 33-\mathrm{O} 5$ & $121.83(17)$ \\
\hline $\mathrm{Co} 2-\mathrm{O} 13-\mathrm{H} 26$ & $103(2)$ & $\mathrm{O} 4-\mathrm{C} 33-\mathrm{C} 34$ & $120.09(17)$ \\
\hline $\mathrm{Co} 2-\mathrm{O} 13-\mathrm{H} 27$ & $123.7(19)$ & $\mathrm{O} 5-\mathrm{C} 33-\mathrm{C} 34$ & $118.09(16)$ \\
\hline $\mathrm{H} 26-\mathrm{O} 13-\mathrm{H} 27$ & $117(3)$ & $\mathrm{C} 35-\mathrm{C} 34-\mathrm{C} 33$ & $122.02(17)$ \\
\hline $\mathrm{C} 1-\mathrm{N} 1-\mathrm{Co} 1$ & $122.34(13)$ & C39-C34-C33 & $119.26(17)$ \\
\hline $\mathrm{C} 5-\mathrm{N} 1-\mathrm{Co} 1$ & $121.01(13)$ & C39-C34-C35 & $118.67(18)$ \\
\hline $\mathrm{C} 5-\mathrm{N} 1-\mathrm{C} 1$ & $116.64(17)$ & $\mathrm{O} 6-\mathrm{C} 35-\mathrm{C} 34$ & $122.54(18)$ \\
\hline $\mathrm{C} 10-\mathrm{N} 2-\mathrm{C} 6$ & $115.94(19)$ & $\mathrm{O} 6-\mathrm{C} 35-\mathrm{C} 36$ & $117.85(19)$ \\
\hline $\mathrm{C} 11-\mathrm{N} 3-\mathrm{Co} 1$ & $121.43(13)$ & $\mathrm{C} 36-\mathrm{C} 35-\mathrm{C} 34$ & $119.59(19)$ \\
\hline $\mathrm{C} 15-\mathrm{N} 3-\mathrm{Co} 1$ & $121.58(13)$ & C $35-\mathrm{C} 36-\mathrm{H} 36$ & 119.9 \\
\hline $\mathrm{C} 15-\mathrm{N} 3-\mathrm{C} 11$ & $116.95(16)$ & $\mathrm{C} 37-\mathrm{C} 36-\mathrm{C} 35$ & $120.1(2)$ \\
\hline $\mathrm{C} 16-\mathrm{N} 4-\mathrm{Co} 2$ & $124.46(13)$ & С $37-\mathrm{C} 36-\mathrm{H} 36$ & 119.9 \\
\hline $\mathrm{C} 16-\mathrm{N} 4-\mathrm{C} 20$ & $116.83(17)$ & C36-C37- H37 & 119.5 \\
\hline $\mathrm{C} 20-\mathrm{N} 4-\mathrm{Co} 2$ & $118.69(13)$ & $\mathrm{C} 36-\mathrm{C} 37-\mathrm{C} 38$ & $121.1(2)$ \\
\hline $\mathrm{C} 21-\mathrm{N} 5-\mathrm{Co} 2$ & $123.24(14)$ & C38-C37-H37 & 119.5 \\
\hline
\end{tabular}




\begin{tabular}{|c|c|c|c|}
\hline $\mathrm{C} 21-\mathrm{N} 5-\mathrm{C} 25$ & $116.75(18)$ & $\mathrm{C} 37-\mathrm{C} 38-\mathrm{H} 38$ & 120.4 \\
\hline $\mathrm{C} 25-\mathrm{N} 5-\mathrm{Co} 2$ & 119.69 (14) & $\mathrm{C} 37-\mathrm{C} 38-\mathrm{C} 39$ & $119.2(2)$ \\
\hline $\mathrm{N} 1-\mathrm{C} 1-\mathrm{H} 1$ & 118.4 & $\mathrm{C} 39-\mathrm{C} 38-\mathrm{H} 38$ & 120.4 \\
\hline $\mathrm{N} 1-\mathrm{C} 1-\mathrm{C} 2$ & $123.22(19)$ & $\mathrm{C} 34-\mathrm{C} 39-\mathrm{H} 39$ & 119.3 \\
\hline $\mathrm{C} 2-\mathrm{C} 1-\mathrm{H} 1$ & 118.4 & $\mathrm{C} 38-\mathrm{C} 39-\mathrm{C} 34$ & $121.3(2)$ \\
\hline $\mathrm{C} 1-\mathrm{C} 2-\mathrm{H} 2$ & 119.8 & $\mathrm{C} 38-\mathrm{C} 39-\mathrm{H} 39$ & 119.3 \\
\hline $\mathrm{C} 1-\mathrm{C} 2-\mathrm{C} 3$ & $120.44(19)$ & $\mathrm{O} 7-\mathrm{C} 40-\mathrm{Co} 2$ & $62.59(11)$ \\
\hline $\mathrm{C} 3-\mathrm{C} 2-\mathrm{H} 2$ & 119.8 & $\mathrm{O} 7-\mathrm{C} 40-\mathrm{C} 41$ & $119.59(18)$ \\
\hline $\mathrm{C} 2-\mathrm{C} 3-\mathrm{C} 4$ & $116.31(18)$ & $\mathrm{O} 8-\mathrm{C} 40-\mathrm{Co} 2$ & $57.09(11)$ \\
\hline $\mathrm{C} 2-\mathrm{C} 3-\mathrm{C} 8$ & $121.21(18)$ & $\mathrm{O} 8-\mathrm{C} 40-\mathrm{O} 7$ & $119.58(19)$ \\
\hline $\mathrm{C} 4-\mathrm{C} 3-\mathrm{C} 8$ & $122.48(18)$ & $\mathrm{O} 8-\mathrm{C} 40-\mathrm{C} 41$ & $120.83(19)$ \\
\hline $\mathrm{C} 3-\mathrm{C} 4-\mathrm{H} 4$ & 120.0 & $\mathrm{C} 41-\mathrm{C} 40-\mathrm{Co} 2$ & $176.31(14)$ \\
\hline $\mathrm{C} 5-\mathrm{C} 4-\mathrm{C} 3$ & $119.91(19)$ & $\mathrm{C} 42-\mathrm{C} 41-\mathrm{C} 40$ & $121.4(2)$ \\
\hline $\mathrm{C} 5-\mathrm{C} 4-\mathrm{H} 4$ & 120.0 & $\mathrm{C} 46-\mathrm{C} 41-\mathrm{C} 40$ & $120.0(2)$ \\
\hline $\mathrm{N} 1-\mathrm{C} 5-\mathrm{C} 4$ & $123.47(18)$ & $\mathrm{C} 46-\mathrm{C} 41-\mathrm{C} 42$ & $118.6(2)$ \\
\hline $\mathrm{N} 1-\mathrm{C} 5-\mathrm{H} 5$ & 118.3 & $\mathrm{O} 9-\mathrm{C} 42-\mathrm{C} 41$ & $121.9(2)$ \\
\hline $\mathrm{C} 4-\mathrm{C} 5-\mathrm{H} 5$ & 118.3 & $\mathrm{O} 9-\mathrm{C} 42-\mathrm{C} 43$ & $118.2(3)$ \\
\hline $\mathrm{N} 2-\mathrm{C} 6-\mathrm{H} 6$ & 118.0 & $\mathrm{C} 43-\mathrm{C} 42-\mathrm{C} 41$ & $120.0(3)$ \\
\hline $\mathrm{N} 2-\mathrm{C} 6-\mathrm{C} 7$ & $124.0(2)$ & $\mathrm{C} 42-\mathrm{C} 43-\mathrm{H} 43$ & 120.0 \\
\hline $\mathrm{C} 7-\mathrm{C} 6-\mathrm{H} 6$ & 118.0 & $\mathrm{C} 44-\mathrm{C} 43-\mathrm{C} 42$ & $120.0(3)$ \\
\hline $\mathrm{C} 6-\mathrm{C} 7-\mathrm{H} 7$ & 120.1 & $\mathrm{C} 44-\mathrm{C} 43-\mathrm{H} 43$ & 120.0 \\
\hline $\mathrm{C} 6-\mathrm{C} 7-\mathrm{C} 8$ & $119.8(2)$ & $\mathrm{C} 43-\mathrm{C} 44-\mathrm{H} 44$ & 119.4 \\
\hline $\mathrm{C} 8-\mathrm{C} 7-\mathrm{H} 7$ & 120.1 & $\mathrm{C} 43-\mathrm{C} 44-\mathrm{C} 45$ & $121.1(3)$ \\
\hline $\mathrm{C} 7-\mathrm{C} 8-\mathrm{C} 3$ & $121.04(18)$ & $\mathrm{C} 45-\mathrm{C} 44-\mathrm{H} 44$ & 119.4 \\
\hline $\mathrm{C} 9-\mathrm{C} 8-\mathrm{C} 3$ & $122.60(18)$ & $\mathrm{C} 44-\mathrm{C} 45-\mathrm{H} 45$ & 120.3 \\
\hline $\mathrm{C} 9-\mathrm{C} 8-\mathrm{C} 7$ & $116.33(19)$ & $\mathrm{C} 44-\mathrm{C} 45-\mathrm{C} 46$ & $119.5(3)$ \\
\hline $\mathrm{C} 8-\mathrm{C} 9-\mathrm{H} 9 \mathrm{~A}$ & 120.1 & $\mathrm{C} 46-\mathrm{C} 45-\mathrm{H} 45$ & 120.3 \\
\hline $\mathrm{C} 10-\mathrm{C} 9-\mathrm{C} 8$ & $119.8(2)$ & $\mathrm{C} 41-\mathrm{C} 46-\mathrm{H} 46$ & 119.6 \\
\hline $\mathrm{C} 10-\mathrm{C} 9-\mathrm{H} 9 \mathrm{~A}$ & 120.1 & $\mathrm{C} 45-\mathrm{C} 46-\mathrm{C} 41$ & $120.8(3)$ \\
\hline $\mathrm{N} 2-\mathrm{C} 10-\mathrm{C} 9$ & $124.1(2)$ & $\mathrm{C} 45-\mathrm{C} 46-\mathrm{H} 46$ & 119.6 \\
\hline $\mathrm{N} 2-\mathrm{C} 10-\mathrm{H} 10$ & 118.0 & $\mathrm{O} 10-\mathrm{C} 47-\mathrm{O} 11$ & $124.1(2)$ \\
\hline $\mathrm{C} 9-\mathrm{C} 10-\mathrm{H} 10$ & 118.0 & $\mathrm{O} 10-\mathrm{C} 47-\mathrm{C} 48$ & $118.1(2)$ \\
\hline $\mathrm{N} 3-\mathrm{C} 11-\mathrm{H} 11$ & 118.4 & $\mathrm{O} 11-\mathrm{C} 47-\mathrm{C} 48$ & 117.77 (19) \\
\hline $\mathrm{N} 3-\mathrm{C} 11-\mathrm{C} 12$ & $123.28(18)$ & $\mathrm{C} 49-\mathrm{C} 48-\mathrm{C} 47$ & $121.1(2)$ \\
\hline $\mathrm{C} 12-\mathrm{C} 11-\mathrm{H} 11$ & 118.4 & $\mathrm{C} 53-\mathrm{C} 48-\mathrm{C} 47$ & $120.76(19)$ \\
\hline $\mathrm{C} 11-\mathrm{C} 12-\mathrm{H} 12$ & 120.0 & $\mathrm{C} 53-\mathrm{C} 48-\mathrm{C} 49$ & $118.1(2)$ \\
\hline $\mathrm{C} 11-\mathrm{C} 12-\mathrm{C} 13$ & $120.09(18)$ & $\mathrm{O} 12-\mathrm{C} 49-\mathrm{C} 48$ & $121.4(2)$ \\
\hline $\mathrm{C} 13-\mathrm{C} 12-\mathrm{H} 12$ & 120.0 & $\mathrm{O} 12-\mathrm{C} 49-\mathrm{C} 50$ & $118.5(2)$ \\
\hline $\mathrm{C} 12-\mathrm{C} 13-\mathrm{C} 14$ & $116.58(17)$ & $\mathrm{C} 50-\mathrm{C} 49-\mathrm{C} 48$ & $120.1(2)$ \\
\hline $\mathrm{C} 12-\mathrm{C} 13-\mathrm{C} 18$ & $121.10(17)$ & $\mathrm{C} 49-\mathrm{C} 50-\mathrm{H} 50$ & 119.9 \\
\hline $\mathrm{C} 14-\mathrm{C} 13-\mathrm{C} 18$ & $122.32(17)$ & $\mathrm{C} 51-\mathrm{C} 50-\mathrm{C} 49$ & $120.2(2)$ \\
\hline $\mathrm{C} 13-\mathrm{C} 14-\mathrm{H} 14$ & 120.1 & $\mathrm{C} 51-\mathrm{C} 50-\mathrm{H} 50$ & 119.9 \\
\hline $\mathrm{C} 15-\mathrm{C} 14-\mathrm{C} 13$ & $119.89(17)$ & $\mathrm{C} 50-\mathrm{C} 51-\mathrm{H} 51$ & 119.9 \\
\hline $\mathrm{C} 15-\mathrm{C} 14-\mathrm{H} 14$ & 120.1 & $\mathrm{C} 50-\mathrm{C} 51-\mathrm{C} 52$ & $120.3(2)$ \\
\hline $\mathrm{N} 3-\mathrm{C} 15-\mathrm{C} 14$ & $123.20(18)$ & $\mathrm{C} 52-\mathrm{C} 51-\mathrm{H} 51$ & 119.9 \\
\hline $\mathrm{N} 3-\mathrm{C} 15-\mathrm{H} 15$ & 118.4 & $\mathrm{C} 51-\mathrm{C} 52-\mathrm{H} 52$ & 120.0 \\
\hline $\mathrm{C} 14-\mathrm{C} 15-\mathrm{H} 15$ & 118.4 & $\mathrm{C} 53-\mathrm{C} 52-\mathrm{C} 51$ & $120.0(2)$ \\
\hline $\mathrm{N} 4-\mathrm{C} 16-\mathrm{H} 16$ & 118.3 & $\mathrm{C} 53-\mathrm{C} 52-\mathrm{H} 52$ & 120.0 \\
\hline
\end{tabular}


N4-C16-C17

$\mathrm{C} 17-\mathrm{C} 16-\mathrm{H} 16$

$\mathrm{C} 16-\mathrm{C} 17-\mathrm{H} 17$

$\mathrm{C} 16-\mathrm{C} 17-\mathrm{C} 18$

$\mathrm{Co} 1-\mathrm{O} 1-\mathrm{C} 26-\mathrm{O} 2$

$\mathrm{Co} 1-\mathrm{O} 1-\mathrm{C} 26-\mathrm{C} 27$

$\mathrm{Co} 1-\mathrm{O} 2-\mathrm{C} 26-\mathrm{O} 1$

$\mathrm{Co} 1-\mathrm{O} 2-\mathrm{C} 26-\mathrm{C} 27$

$\mathrm{Co} 1-\mathrm{O} 4-\mathrm{C} 33-\mathrm{O} 5$

$\mathrm{Co} 1-\mathrm{O} 4-\mathrm{C} 33-\mathrm{C} 34$

$\mathrm{Co} 1-\mathrm{O} 5-\mathrm{C} 33-\mathrm{O} 4$

$\mathrm{Co} 1-\mathrm{O} 5-\mathrm{C} 33-\mathrm{C} 34$

$\mathrm{Co} 1-\mathrm{N} 1-\mathrm{C} 1-\mathrm{C} 2$

Co1-N1-C5-C4

$\mathrm{Co} 1-\mathrm{N} 3-\mathrm{C} 11-\mathrm{C} 12$

$\mathrm{Co} 1-\mathrm{N} 3-\mathrm{C} 15-\mathrm{C} 14$

$\mathrm{Co} 2-\mathrm{O} 7-\mathrm{C} 40-\mathrm{O} 8$

$\mathrm{Co} 2-\mathrm{O} 7-\mathrm{C} 40-\mathrm{C} 41$

$\mathrm{Co} 2-\mathrm{O} 8-\mathrm{C} 40-\mathrm{O} 7$

$\mathrm{Co} 2-\mathrm{O} 8-\mathrm{C} 40-\mathrm{C} 41$

$\mathrm{Co} 2-\mathrm{O} 10-\mathrm{C} 47-\mathrm{O} 11$

$\mathrm{Co} 2-\mathrm{O} 10-\mathrm{C} 47-\mathrm{C} 48$

$\mathrm{Co} 2-\mathrm{N} 4-\mathrm{C} 16-\mathrm{C} 17$

$\mathrm{Co} 2-\mathrm{N} 4-\mathrm{C} 20-\mathrm{C} 19$

Co2-N5-C21-C22

$\mathrm{Co} 2-\mathrm{N} 5-\mathrm{C} 25-\mathrm{C} 24$

$\mathrm{O} 1-\mathrm{C} 26-\mathrm{C} 27-\mathrm{C} 28$

$\mathrm{O} 1-\mathrm{C} 26-\mathrm{C} 27-\mathrm{C} 32$

$\mathrm{O} 2-\mathrm{C} 26-\mathrm{C} 27-\mathrm{C} 28$

$\mathrm{O} 2-\mathrm{C} 26-\mathrm{C} 27-\mathrm{C} 32$

$\mathrm{O} 3-\mathrm{C} 28-\mathrm{C} 29-\mathrm{C} 30$

$\mathrm{O} 4-\mathrm{C} 33-\mathrm{C} 34-\mathrm{C} 35$

$\mathrm{O} 4-\mathrm{C} 33-\mathrm{C} 34-\mathrm{C} 39$

$\mathrm{O} 5-\mathrm{C} 33-\mathrm{C} 34-\mathrm{C} 35$

$\mathrm{O} 5-\mathrm{C} 33-\mathrm{C} 34-\mathrm{C} 39$

$\mathrm{O} 6-\mathrm{C} 35-\mathrm{C} 36-\mathrm{C} 37$

$\mathrm{O} 7-\mathrm{C} 40-\mathrm{C} 41-\mathrm{C} 42$

$\mathrm{O} 7-\mathrm{C} 40-\mathrm{C} 41-\mathrm{C} 46$

$\mathrm{O} 8-\mathrm{C} 40-\mathrm{C} 41-\mathrm{C} 42$

$\mathrm{O} 8-\mathrm{C} 40-\mathrm{C} 41-\mathrm{C} 46$

$\mathrm{O} 9-\mathrm{C} 42-\mathrm{C} 43-\mathrm{C} 44$

$\mathrm{O} 10-\mathrm{C} 47-\mathrm{C} 48-\mathrm{C} 49$

$\mathrm{O} 10-\mathrm{C} 47-\mathrm{C} 48-\mathrm{C} 53$

$\mathrm{O} 11-\mathrm{C} 47-\mathrm{C} 48-\mathrm{C} 49$

$\mathrm{O} 11-\mathrm{C} 47-\mathrm{C} 48-\mathrm{C} 53$

$\mathrm{O} 12-\mathrm{C} 49-\mathrm{C} 50-\mathrm{C} 51$

$\mathrm{N} 1-\mathrm{C} 1-\mathrm{C} 2-\mathrm{C} 3$
$123.43(18)$

118.3

120.1

$119.76(18)$

$1.3(2)$

$-178.48(17)$

$-1.3(2)$

$178.48(18)$

$98.2(6)$

$-81.8(6)$

$0.1(2)$

$-179.90(12)$

$-178.47(17)$

$178.93(16)$

$-178.99(16)$

$178.79(16)$

$3.61(18)$

$-176.61(17)$

$-3.81(19)$

$176.41(16)$

$13.9(3)$

$-166.89(13)$

$179.12(15)$

$-179.40(16)$

$-172.7(2)$

$172.38(17)$

$6.0(3)$

$-173.9(2)$

$-173.8(2)$

$6.4(3)$

$-177.5(3)$

$178.91(18)$

$1.4(3)$

$-1.0(3)$

$-178.55(18)$

$-179.0(2)$

$-2.6(3)$

$178.6(2)$

$177.2(2)$

-1.7 (3)

$179.5(4)$

$-175.88(19)$

$4.6(3)$

$3.4(3)$

$-176.1(2)$

$179.4(2)$

$-0.8(3)$
$\mathrm{C} 48-\mathrm{C} 53-\mathrm{H} 53$

$\mathrm{C} 52-\mathrm{C} 53-\mathrm{C} 48$

$\mathrm{C} 52-\mathrm{C} 53-\mathrm{H} 53$

$\mathrm{C} 11-\mathrm{N} 3-\mathrm{C} 15-\mathrm{C} 14$

$\mathrm{C} 11-\mathrm{C} 12-\mathrm{C} 13-\mathrm{C} 14$

$\mathrm{C} 11-\mathrm{C} 12-\mathrm{C} 13-\mathrm{C} 18$

$\mathrm{C} 12-\mathrm{C} 13-\mathrm{C} 14-\mathrm{C} 15$

$\mathrm{C} 12-\mathrm{C} 13-\mathrm{C} 18-\mathrm{C} 17$

$\mathrm{C} 12-\mathrm{C} 13-\mathrm{C} 18-\mathrm{C} 19$

$\mathrm{C} 13-\mathrm{C} 14-\mathrm{C} 15-\mathrm{N} 3$

$\mathrm{C} 13-\mathrm{C} 18-\mathrm{C} 19-\mathrm{C} 20$

$\mathrm{C} 14-\mathrm{C} 13-\mathrm{C} 18-\mathrm{C} 17$

$\mathrm{C} 14-\mathrm{C} 13-\mathrm{C} 18-\mathrm{C} 19$

$\mathrm{C} 15-\mathrm{N} 3-\mathrm{C} 11-\mathrm{C} 12$

$\mathrm{C} 16-\mathrm{N} 4-\mathrm{C} 20-\mathrm{C} 19$

C16-C17-C18-C13

C16-C17-C18-C19

$\mathrm{C} 17-\mathrm{C} 18-\mathrm{C} 19-\mathrm{C} 20$

$\mathrm{C} 18-\mathrm{C} 13-\mathrm{C} 14-\mathrm{C} 15$

$\mathrm{C} 18-\mathrm{C} 19-\mathrm{C} 20-\mathrm{N} 4$

$\mathrm{C} 20-\mathrm{N} 4-\mathrm{C} 16-\mathrm{C} 17$

$\mathrm{C} 21-\mathrm{N} 5-\mathrm{C} 25-\mathrm{C} 24$

$\mathrm{C} 21-\mathrm{C} 22-\mathrm{C} 23-\mathrm{C} 23^{\mathrm{ii}}$

$\mathrm{C} 21-\mathrm{C} 22-\mathrm{C} 23-\mathrm{C} 24$

$\mathrm{C} 22-\mathrm{C} 23-\mathrm{C} 24-\mathrm{C} 25$

$\mathrm{C} 23^{\mathrm{ii}}-\mathrm{C} 23-\mathrm{C} 24-\mathrm{C} 25$

$\mathrm{C} 23-\mathrm{C} 24-\mathrm{C} 25-\mathrm{N} 5$

$\mathrm{C} 25-\mathrm{N} 5-\mathrm{C} 21-\mathrm{C} 22$

$\mathrm{C} 26-\mathrm{C} 27-\mathrm{C} 28-\mathrm{O} 3$

$\mathrm{C} 26-\mathrm{C} 27-\mathrm{C} 28-\mathrm{C} 29$

$\mathrm{C} 26-\mathrm{C} 27-\mathrm{C} 32-\mathrm{C} 31$

$\mathrm{C} 27-\mathrm{C} 28-\mathrm{C} 29-\mathrm{C} 30$

$\mathrm{C} 28-\mathrm{C} 27-\mathrm{C} 32-\mathrm{C} 31$

$\mathrm{C} 28-\mathrm{C} 29-\mathrm{C} 30-\mathrm{C} 31$

$\mathrm{C} 29-\mathrm{C} 30-\mathrm{C} 31-\mathrm{C} 32$

$\mathrm{C} 30-\mathrm{C} 31-\mathrm{C} 32-\mathrm{C} 27$

$\mathrm{C} 32-\mathrm{C} 27-\mathrm{C} 28-\mathrm{O} 3$

$\mathrm{C} 32-\mathrm{C} 27-\mathrm{C} 28-\mathrm{C} 29$

$\mathrm{C} 33-\mathrm{C} 34-\mathrm{C} 35-\mathrm{O} 6$

$\mathrm{C} 33-\mathrm{C} 34-\mathrm{C} 35-\mathrm{C} 36$

$\mathrm{C} 33-\mathrm{C} 34-\mathrm{C} 39-\mathrm{C} 38$

$\mathrm{C} 34-\mathrm{C} 35-\mathrm{C} 36-\mathrm{C} 37$

$\mathrm{C} 35-\mathrm{C} 34-\mathrm{C} 39-\mathrm{C} 38$

$\mathrm{C} 35-\mathrm{C} 36-\mathrm{C} 37-\mathrm{C} 38$

$\mathrm{C} 36-\mathrm{C} 37-\mathrm{C} 38-\mathrm{C} 39$

$\mathrm{C} 37-\mathrm{C} 38-\mathrm{C} 39-\mathrm{C} 34$
119.3

$121.3(2)$

119.3

$1.1(3)$

$0.4(3)$

$-179.31(19)$

$-0.5(3)$

$-33.3(3)$

$146.4(2)$

-0.2 (3)

$-179.36(18)$

147.0 (2)

$-33.2(3)$

-1.2 (3)

-0.9 (3)

179.20 (18)

$-0.6(3)$

0.4 (3)

179.13 (19)

0.4 (3)

0.8 (3)

$-1.3(3)$

$179.8(3)$

0.0 (4)

$-0.6(3)$

179.7 (2)

1.3 (4)

0.8 (4)

-2.1 (4)

177.7 (3)

$-179.6(3)$

2.7 (5)

0.6 (4)

$-1.0(6)$

-0.9 (6)

$1.1(5)$

177.8 (3)

-2.5 (4)

1.8 (3)

-176.5 (2)

177.4 (2)

-0.6 (4)

$-0.2(3)$

-0.6 (4)

$1.3(4)$

-0.9 (4) 


$\begin{array}{llll}\mathrm{N} 2-\mathrm{C} 6-\mathrm{C} 7-\mathrm{C} 8 & 0.0(3) & \mathrm{C} 39-\mathrm{C} 34-\mathrm{C} 35-\mathrm{O} 6 & 179.3(2) \\ \mathrm{N} 3-\mathrm{C} 11-\mathrm{C} 12-\mathrm{C} 13 & 0.6(3) & \mathrm{C} 39-\mathrm{C} 34-\mathrm{C} 35-\mathrm{C} 36 & 1.0(3) \\ \mathrm{N} 4-\mathrm{C} 16-\mathrm{C} 17-\mathrm{C} 18 & 0.0(3) & \mathrm{C} 40-\mathrm{C} 41-\mathrm{C} 42-\mathrm{O} 9 & 1.0(4) \\ \mathrm{N} 5-\mathrm{C} 21-\mathrm{C} 22-\mathrm{C} 23 & -0.1(5) & \mathrm{C} 40-\mathrm{C} 41-\mathrm{C} 42-\mathrm{C} 43 & -178.2(3) \\ \mathrm{C} 1-\mathrm{N} 1-\mathrm{C} 5-\mathrm{C} 4 & -0.4(3) & \mathrm{C} 40-\mathrm{C} 41-\mathrm{C} 46-\mathrm{C} 45 & 178.8(3) \\ \mathrm{C} 1-\mathrm{C} 2-\mathrm{C} 3-\mathrm{C} 4 & 0.2(3) & \mathrm{C} 41-\mathrm{C} 42-\mathrm{C} 43-\mathrm{C} 44 & -1.3(5) \\ \mathrm{C} 1-\mathrm{C} 2-\mathrm{C} 3-\mathrm{C} 8 & 179.9(2) & \mathrm{C} 42-\mathrm{C} 41-\mathrm{C} 46-\mathrm{C} 45 & -0.1(4) \\ \mathrm{C} 2-\mathrm{C} 3-\mathrm{C} 4-\mathrm{C} 5 & 0.2(3) & \mathrm{C} 42-\mathrm{C} 43-\mathrm{C} 44-\mathrm{C} 45 & 1.3(7) \\ \mathrm{C} 2-\mathrm{C} 3-\mathrm{C} 8-\mathrm{C} 7 & 26.8(3) & \mathrm{C} 43-\mathrm{C} 44-\mathrm{C} 45-\mathrm{C} 46 & -0.8(7) \\ \mathrm{C} 2-\mathrm{C} 3-\mathrm{C} 8-\mathrm{C} 9 & -151.4(2) & \mathrm{C} 44-\mathrm{C} 45-\mathrm{C} 46-\mathrm{C} 41 & 0.2(5) \\ \mathrm{C} 3-\mathrm{C} 4-\mathrm{C} 5-\mathrm{N} 1 & -0.1(3) & \mathrm{C} 46-\mathrm{C} 41-\mathrm{C} 42-\mathrm{O} 9 & 179.9(3) \\ \mathrm{C} 3-\mathrm{C} 8-\mathrm{C} 9-\mathrm{C} 10 & 176.7(2) & \mathrm{C} 46-\mathrm{C} 41-\mathrm{C} 42-\mathrm{C} 43 & 0.7(4) \\ \mathrm{C} 4-\mathrm{C} 3-\mathrm{C} 8-\mathrm{C} 7 & -153.5(2) & \mathrm{C} 47-\mathrm{C} 48-\mathrm{C} 49-\mathrm{O} 12 & 0.8(3) \\ \mathrm{C} 4-\mathrm{C} 3-\mathrm{C} 8-\mathrm{C} 9 & 28.2(3) & \mathrm{C} 47-\mathrm{C} 48-\mathrm{C} 49-\mathrm{C} 50 & -179.83(19) \\ \mathrm{C} 5-\mathrm{N} 1-\mathrm{C} 1-\mathrm{C} 2 & 0.8(3) & \mathrm{C} 47-\mathrm{C} 48-\mathrm{C} 53-\mathrm{C} 52 & 179.7(2) \\ \mathrm{C} 6-\mathrm{N} 2-\mathrm{C} 10-\mathrm{C} 9 & 1.4(4) & \mathrm{C} 48-\mathrm{C} 49-\mathrm{C} 50-\mathrm{C} 51 & 0.1(3) \\ \mathrm{C} 6-\mathrm{C} 7-\mathrm{C} 8-\mathrm{C} 3 & -176.81(19) & \mathrm{C} 49-\mathrm{C} 48-\mathrm{C} 53-\mathrm{C} 52 & 0.2(3) \\ \mathrm{C} 6-\mathrm{C} 7-\mathrm{C} 8-\mathrm{C} 9 & 1.6(3) & \mathrm{C} 49-\mathrm{C} 50-\mathrm{C} 51-\mathrm{C} 52 & 0.3(4) \\ \mathrm{C} 7-\mathrm{C} 8-\mathrm{C} 9-\mathrm{C} 10 & -1.6(3) & \mathrm{C} 50-\mathrm{C} 51-\mathrm{C} 52-\mathrm{C} 53 & -0.4(4) \\ \mathrm{C} 8-\mathrm{C} 3-\mathrm{C} 4-\mathrm{C} 5 & -179.44(19) & \mathrm{C} 51-\mathrm{C} 52-\mathrm{C} 53-\mathrm{C} 48 & 0.2(4) \\ \mathrm{C} 8-\mathrm{C} 9-\mathrm{C} 10-\mathrm{N} 2 & 0.1(4) & \mathrm{C} 53-\mathrm{C} 48-\mathrm{C} 49-\mathrm{O} 12 & -179.7(2) \\ \mathrm{C} 10-\mathrm{N} 2-\mathrm{C} 6-\mathrm{C} 7 & -1.5(3) & \mathrm{C} 53-\mathrm{C} 48-\mathrm{C} 49-\mathrm{C} 50 & -0.3(3)\end{array}$

Symmetry codes: (i) $-x+1,-y,-z+1$; (ii) $-x+1,-y+2,-z$.

Hydrogen-bond geometry $\left(\AA,{ }^{\circ}\right)$

$C g 4, C g 7$ and $C g 9$ are the centroids of the N4/C16-C20, C34-C39 and C48-C53 rings, respectively.

\begin{tabular}{lllll}
\hline$D-\mathrm{H} \cdots A$ & $D-\mathrm{H}$ & $\mathrm{H} \cdots A$ & $D \cdots A$ & $D-\mathrm{H} \cdots A$ \\
\hline $\mathrm{O} 3-\mathrm{H} 3 \cdots \mathrm{O} 1$ & $0.85(3)$ & $1.77(3)$ & $2.553(3)$ & $153(4)$ \\
$\mathrm{O} 6-\mathrm{H} 8 \cdots \mathrm{O} 5$ & $0.84(2)$ & $1.86(2)$ & $2.587(2)$ & $145(2)$ \\
$\mathrm{O} 9-\mathrm{H} 13 \cdots \mathrm{O} 7$ & $0.84(2)$ & $1.79(2)$ & $2.568(3)$ & $152(4)$ \\
$\mathrm{O} 12-\mathrm{H} 18 \cdots \mathrm{O} 11$ & $0.85(2)$ & $1.74(2)$ & $2.531(2)$ & $154(3)$ \\
$\mathrm{O} 13-\mathrm{H} 26 \cdots \mathrm{O} 11$ & $0.84(1)$ & $1.82(2)$ & $2.625(2)$ & $160(2)$ \\
$\mathrm{O} 13-\mathrm{H} 27 \cdots \mathrm{N} 2^{\mathrm{i}}$ & $0.83(2)$ & $2.05(2)$ & $2.860(3)$ & $167(2)$ \\
$\mathrm{C} 25-\mathrm{H} 25 \cdots \mathrm{O} 8$ & 0.93 & 2.55 & $3.143(3)$ & 122 \\
$\mathrm{C} 39-\mathrm{H} 39 \cdots \mathrm{O} 1$ & 0.93 & 2.38 & $3.271(3)$ & 160 \\
$\mathrm{C} 5-\mathrm{H} 5 \cdots \mathrm{O} 12^{\mathrm{iii}}$ & 0.93 & 2.59 & $3.324(3)$ & 136 \\
$\mathrm{C} 6-\mathrm{H} 6 \cdots \mathrm{O} 7^{\mathrm{iv}}$ & 0.93 & 2.48 & $3.300(3)$ & 147 \\
$\mathrm{C} 12-\mathrm{H} 12 \cdots \mathrm{O} 3^{\mathrm{v}}$ & 0.93 & 2.58 & $3.173(3)$ & 122 \\
$\mathrm{C} 15-\mathrm{H} 15 \cdots \mathrm{O} 6^{\text {vi }}$ & 0.93 & 2.51 & $3.292(3)$ & 142 \\
$\mathrm{C} 2-\mathrm{H} 2 \cdots C g 9^{\text {iv }}$ & 0.93 & 2.95 & $3.833(2)$ & 160 \\
$\mathrm{C} 31-\mathrm{H} 31 \cdots C g 4^{\text {vii }}$ & 0.93 & 2.85 & $3.681(4)$ & 149 \\
$\mathrm{C} 51-\mathrm{H} 51 \cdots C g 7^{\text {viii }}$ & 0.93 & 2.72 & $3.623(3)$ & 165 \\
\hline
\end{tabular}

Symmetry codes: (i) $-x+1,-y,-z+1$; (iii) $x, y-1, z+1$; (iv) $x+1, y-1, z+1$; (v) $-x+1,-y+1,-z+1$; (vi) $x-1, y, z$; (vii) $-x,-y+1,-z+1$; (viii) $x-1, y+1, z-1$. 